Article

\title{
The Non-Equilibrium Statistical Distribution Function for Electrons and Holes in Semiconductor Heterostructures in Steady-State Conditions
}

\author{
Krzysztof Jóźwikowski $^{1, *}$, Alina Jóźwikowska ${ }^{2}$ and Michał Nietopiel ${ }^{1}$ \\ ${ }^{1}$ Institute of Applied Physics, Military University of Technology, 2 Kaliskiego St., 00-908 Warsaw, \\ Poland; E-Mail: nietem@poczta.onet.pl \\ ${ }^{2}$ Faculty of Applied Informatics and Mathematics, Warsaw University of Life Science SGGW, \\ Nowoursynowska 166 St., 02-787 Warsaw, Poland; E-Mail: alina_jozwikowska@sggw.pl \\ * Author to whom correspondence should be addressed; E-Mail: kjozwikowski@wat.edu.pl; \\ Tel.: +48-22-683-91-09.
}

Academic Editor: Kevin H. Knuth

Received: 6 April 2015 / Accepted: 10 June 2015 / Published: 15 June 2015

\begin{abstract}
The main goal of this work is to determine a statistical non-equilibrium distribution function for the electron and holes in semiconductor heterostructures in steady-state conditions. Based on the postulates of local equilibrium, as well as on the integral form of the weighted Gyarmati's variational principle in the force representation, using an alternative method, we have derived general expressions, which have the form of the Fermi-Dirac distribution function with four additional components. The physical interpretation of these components has been carried out in this paper. Some numerical results of a non-equilibrium distribution function for an electron in $\mathrm{HgCdTe}$ structures are also presented.
\end{abstract}

Keywords: non-equilibrium distribution function; semiconductor heterostructure; postulate of local equilibrium; entropy generation; Gyarmati's principle; $\mathrm{HgCdTe}$ structures

PACS classifications: B15:3 


\section{Introduction}

In order to completely specify the operation of a device, we should ask what is the probability of finding a carrier with crystal momentum $\vec{k}$ at location $\vec{r}$ at time $t$ ? The answer is the distribution function $f=f(\vec{k}, \vec{r}, t)$, a number between zero and one. The distribution function describes an average distribution of carriers in both position and momentum and can be used to obtain various quantities of interest, such as carrier, current and kinetic energy densities. Progress in molecular beam epitaxy (MBE) and metal organic chemical vapor deposition (MOCVD) epitaxial techniques makes it possible to fabricate heterostructures used to manufacture numerous new devices, such as two-color photodiodes matrices, super lattices, quantum dots, etc. The modeling of these devices is based on specialized computer software, whose key task is to calculate the distribution functions mentioned above. The distribution functions describing the statistics of carriers in semiconductor structures in thermal equilibrium usually differ quite significantly from those describing non-equilibrium states. Usually, in order to find a non-equilibrium distribution function for the electron and holes, the Boltzmann transport equation (BTE) is solved with a relaxation time approximation [1-4]. However, the distribution functions derived in this way may be used only in the cases where gradients of electrochemical potentials and gradients of temperature are sufficiently low [5]. By coupling the basic postulate of the thermodynamics of irreversible processes referring to entropy generation with the weighted Gyarmati's principle in the forces representation, we have derived functionals adopting their extreme values in a steady state. Euler-Lagrange equations formulated for these functionals enable the determination of the distribution function for an electron in a conduction band (CB) and in a valence band (VB), which may be used in the case where strong gradients of temperature and those of quasi-Fermi energies occur.

\section{Irreversible Process}

The basic postulate of the thermodynamics of irreversible processes refers to the entropy generation. As a result of the irreversible process proceeding in the element of volume $d V$ entropy generation is obtained with the rate of $\sigma_{S} d V . \sigma_{S} \geq 0$, which is always a non-negative entropy generation rate (in unit volume and unit time), which equals zero only in equilibrium. The change of entropy in the given element $d V$ in the time period $d t$ is, however, the result of not only the entropy generation in the element itself, but it is also caused by an exchange of heat energy with its environment. Thus, it could be written as:

$$
d s=\sigma_{S} d t+\frac{d q}{T}
$$

where $d s$ is the difference of entropy density, $T$ is the temperature and $d q$ is the difference of heat density, which is exchanged by the element $d V$ with its environment. Since there is always $\sigma_{S} \geq 0$, therefore:

$$
d s \geq \frac{d q}{T}
$$

Equality in relation Equation (2) refers to reversible processes and non-equality to irreversible processes. In a system being in non-equilibrium conditions, gradients of intense state parameters (gradients of temperature and electrochemical potentials) occur. In semiconductors, relation Equation (2) may be referred to as the selected kinds of charge carriers, which are treated as a system. We can 
distinguish, for instance, between electrons in a conduction band and holes in a valence band, ionized impurities, etc. For the $i$-type particles, relation Equation (2) reads as:

$$
d s_{i} \geq \frac{d q_{i}}{T_{i}}
$$

The steady state is the goal of our considerations. In classical irreversible thermodynamics [6-9], it is presumed that small elements of a non-equilibrium system are in a state of local equilibrium, and equations of equilibrium thermodynamics hold well for such sub-system. This postulate is known as a local thermodynamic equilibrium.

Thus, considering the state of a small sub-volume $d V$ of the semiconductor heterostructure, one can apply Gibbs relation to express the differential of heat density $d q_{i}$ of $i$-type particles by the differential of density of their energy $d u_{i}$, as well as the differential of particle density $d n_{i}$. Then, inequality Equation (2a) assumes the relation as:

$$
d s_{i}-\frac{d u_{i}}{T_{i}}+\frac{\Phi_{i}}{T_{i}} d n_{i} \geq 0
$$

where $\Phi_{i}$ is the electrochemical potential of $i$-type particles (for example, electrons in a conduction band). In a steady state, both intense and extensive state parameters are stable in an arbitrarily chosen element of the volume $d V$. Due to relation Equation (3), the function:

$$
m_{i}=s_{i}-\frac{u_{i}}{T_{i}}+\frac{\Phi_{i}}{T_{i}} n_{i}
$$

which is determined in $d V$ should achieve maximum values in a steady state.

In a steady state, a source of entropy generates an entropy in each cell of space with a possible low rate. This is in accordance with Prigogine principle [10,11]. If we denote the efficiency of the entropy source (in $\mathrm{J} \mathrm{K}^{-1} \mathrm{~cm}^{-3}$ ) for $i$-kind particles as $\sigma_{S_{i}}$, then in steady-state conditions:

$$
\sigma_{S_{i}} \geq 0 \quad \text { and } \quad \sigma_{S_{i}}=\min
$$

The steady state being a non-equilibrium state is caused by stationary gradients of state intensive parameters (temperature and electrochemical potentials, usually called quasi-Fermi energies), which cause flows of energy and electric carriers. However, these flows take place in a very complex and complicated way, being the result of a huge number of single acts of an electron and phonon scattering in a dissipative process. The integration of relation Equation (3) over all of the volume of the semiconductor structure leads to a relation of:

$$
\int_{V}\left(d s_{i}-\frac{d u_{i}}{T_{i}}+\frac{\Phi_{i}}{T_{i}} d n_{i}\right) d V \geq 0 .
$$

Let us denote as $M_{i}$ the following integral:

$$
M_{i}=\int_{V} m_{i} d V=\int_{V}\left(s_{i}-\frac{u_{i}}{T_{i}}+\frac{\Phi_{i}}{T_{i}} n_{i}\right) d V \geq 0 .
$$

Thus, the right side of Equation (6) is the differential $d M_{i}$ of the function $M_{i}$. Since $d M_{i}$ is always positive during the irreversible process, then, in the steady state, $M_{i}$ being of stable quantity achieves its maximum value. This value is stable as long as the steady state lasts (see Figure 1). 
Thus, in a steady state, the condition for the maximum of the following functional can be formulated as:

$$
M_{i}=\int_{V}\left(s_{i}-\frac{u_{i}}{T_{i}}+\frac{\Phi_{i}}{T_{i}} n_{i}\right) d V=\max
$$

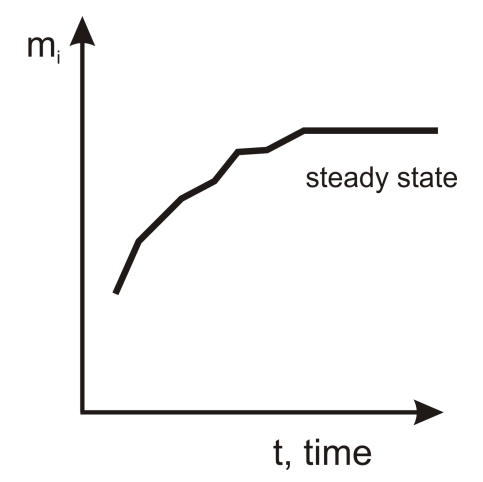

Figure 1. In an irreversible process, differentials of the function $m_{i}$ in an arbitrarily chosen element of volume are always positive. This is the reason why in the steady state, $m_{i}$ achieves its maximum values.

The state of intense parameters contained in functional Equation (8) are space-dependent quantities; however, they do not change in time. Using the expressions for electron and hole energy, as well as for their entropy (Equations (46)-(49)), the function for the electron and holes can be derived as below:

$$
\begin{aligned}
m_{e}= & k_{B} \frac{1}{4 \pi^{3}} \int_{B Z}\left\{f_{e}^{C}(\vec{r}, \vec{k}) \ln f_{e}^{C}(\vec{r}, \vec{k})+\left[1-f_{e}^{C}(\vec{r}, \vec{k})\right] \ln \left[1-f_{e}^{C}(\vec{r}, \vec{k})\right]\right\} d^{3} k \\
& +\frac{1}{4 \pi^{3}} \int_{B Z} f_{e}^{C}(\vec{r}, \vec{k})\left(\chi(\vec{r})+e \Psi(\vec{r})-\varepsilon_{C}^{k}(\vec{k})+\Phi_{n}\right) \frac{1}{T_{e}(\vec{r})} d^{3} k
\end{aligned}
$$

and:

$$
\begin{aligned}
m_{h}= & k_{B} \frac{1}{4 \pi^{3}} \int_{B Z}\left\{f_{e}^{V}(\vec{r}, \vec{k}) \ln f_{e}^{V}(\vec{r}, \vec{k})+\left[1-f_{e}^{V}(\vec{r}, \vec{k})\right] \ln \left[1-f_{e}^{V}(\vec{r}, \vec{k})\right]\right\} d^{3} k \\
& +\frac{1}{4 \pi^{3}} \int_{B Z}\left[1-f_{e}^{V}(\vec{r}, \vec{k})\right]\left(-\chi(\vec{r})-e \Psi(\vec{r})-E_{g}(\vec{r})-\varepsilon_{V}^{k}(\vec{k})+\Phi_{p}\right) \frac{1}{T_{h}(\vec{r})} d^{3} k
\end{aligned}
$$

respectively. Here, $f_{e}^{C}(\vec{r}, \vec{k})$ is the distribution function for electrons in a conduction band and $f_{e}^{V}(\vec{r}, \vec{k})$ is the distribution function for electrons in a valence band.

Functional Equation (8) for the electron and holes achieves the maximum if:

$$
\frac{\partial m_{e}}{\partial f_{e}^{C}}=0
$$

and:

$$
\frac{\partial m_{h}}{\partial f_{e}^{v}}=0
$$


respectively. Equations (11) and (12) are Euler-Lagrange (E-L) equations, and their solution are the functions:

$$
\begin{aligned}
& f_{C}^{e}(\vec{r}, \vec{k})=\left(1+\exp \left(\frac{-\chi(\vec{r})-e \Psi(\vec{r})+\varepsilon_{C}^{k}(\vec{k})-\Phi_{n}(\vec{r})}{k_{B} T_{e}(\vec{r})}\right)\right)^{-1} \\
& f_{V}^{e}(\vec{r}, \vec{k})=\left(1+\exp \left(\frac{-\chi(\vec{r})-e \Psi(\vec{r})-E_{g}(\vec{r})-\varepsilon_{V}^{k}(\vec{k})-\Phi_{p}(\vec{r})}{k_{B} T_{h}(\vec{r})}\right)\right)^{-1}
\end{aligned}
$$

It is apparent that the above functions cannot be the correct distribution functions by observing that they are even in the wave vector (kinetic energies $\varepsilon_{C}^{k}(\vec{k})$ and $\varepsilon_{V}^{k}(\vec{k})$ are square functions of $\vec{k}$ ), and therefore, it can be predicted that current can never flow. Nevertheless, these are not unreasonable results considering that average carrier velocities are frequently small. If we measure the spread in velocities by the velocity at which $f(\vec{r}, \vec{k})$ drops to $\frac{1}{e}$ of its peak, we find a spread of about $10^{7} \mathrm{~cm} \mathrm{~s}^{-1}$ for a semiconductor with $m^{*} \approx m_{0}$ [12]. Since average velocities are often much smaller than this, an assumption that the average velocity equals zero may be not as bad as it seems. However, we will try to find more correct expressions for the distribution functions.

\section{Gyarmati’s Variational Principle of Dissipative Processes}

Non-equilibrium processes arise either due to the action of thermodynamic forces, which prevent the system from reaching the equilibrium state, or due to the process of internal phenomena, resulting from certain types of relaxation processes [13]. In the 1960s, Gyarmati proposed a variational principle, which describes the evolution of irreversible processes in space and time [14,15]. Gyarmati's principle is based on the fact that the generalization of dissipation functions, which were introduced by Reyleigh and Onsager for special cases, always exist locally in continua [16-22]. In linear theory, these functions are defined as:

$$
\Psi(X)=\frac{1}{2} \sum_{i, k} L_{i k} X_{i} X_{k} \geq 0
$$

and:

$$
\Phi(J)=\frac{1}{2} \sum_{i k} R_{i k} J_{i} J_{k} \geq 0 .
$$

The $R_{i k}$ coefficients are the components of the inverse of the $L_{i k}$ coefficient; hence, the existence of dissipation potentials is connected to the existence of the Onsager reciprocal relations. The current $J_{i}$ is conjugated to the force $X_{i}$ in the entropy production density $\sigma_{S}$. $\sigma_{S}$ being the entropy production per unit volume and unit time is a bilinear function of the independent currents $J_{i}$ and the conjugate dissipative forces $X_{i}$, that is:

$$
\sigma_{S}=\sum_{i} X_{i} J_{i} \geq 0
$$

According to the Onsager's linear theory of irreversible processes, the currents $J_{i}$ and the dissipative forces $X_{i}$ are given by the following general constitutive laws [20,21]:

$$
J_{i}=\sum_{k} L_{i k} X_{i}, \quad X_{i}=\sum_{k} R_{i k} J_{k}
$$




$$
J_{i}=\frac{\partial \Psi}{\partial X_{i}}, \quad X_{i}=\frac{\partial \Phi}{\partial J_{i}} .
$$

The most general form of Gyarmati's principle is given by:

$$
\delta \int_{V}\left[\sigma_{S}-\Psi-\Phi\right] d V=0
$$

or:

$$
\int_{V}\left[\sigma_{S}-\Psi-\Phi\right] d V=\max .
$$

Some weighted potentials $\Psi^{G}$ and $\Phi^{G}$ can be defined, as well. They show all of the essential properties of $\Psi$ and $\Phi$, but correspond to the weighted entropy production $G \sigma_{S}$ [23-25] (here, $G$ is an arbitrary, always positive state function):

$$
G \sigma_{S}=\sum_{i} G X_{i} J_{i} \geq 0
$$

In some cases, in steady-state conditions, especially for strictly linear problems, there are two partial forms that are also valid [25]:

$$
\int_{V}\left[\sigma_{S}-\Psi\right] d V=\max , \quad J=\text { const }
$$

and:

$$
\int_{V}\left[\sigma_{S}-\Phi\right] d V=\max , \quad X=\text { const } .
$$

The first of these is called force, and the second is called flux representation. Both representations were applied to the solution of several practical problems (see the references cited in the paper of Verhas [25]).

We will apply Equation (23) for electrons and holes. The thermodynamic forces for electrons (see relation Equation (72)) are recognized as:

$$
X_{1}=\frac{\nabla \Phi_{n}(\vec{r})}{T_{e}(\vec{r})} ; \quad X_{2}=\frac{\nabla T_{e}(\vec{r})}{T_{e}(\vec{r})}
$$

and:

$$
\Psi(X)=\frac{1}{2}\left(L_{11} X_{1} X_{1}+L_{12} X_{1} X_{2}+L_{21} X_{2} X_{1}+L_{22} X_{2} X_{2}\right)
$$

where:

$$
\begin{aligned}
L_{11}= & \frac{T_{e}(\vec{r})}{4 \pi^{3}} \int_{B Z} f_{e}^{C}(\vec{r}, \vec{k}) \frac{\bar{\tau}_{e}}{m_{e}^{*}} d^{3} k \\
L_{12}= & L_{21}=-\frac{k_{B} T_{e}(\vec{r})}{4 \pi^{3}} \int_{B Z}\left\{f_{e}^{C}(\vec{r}, \vec{k}) \ln f_{e}^{C}(\vec{r}, \vec{k})+\left[1-f_{e}^{C}(\vec{r}, \vec{k})\right] \ln \left[1-f_{e}^{C}(\vec{r}, \vec{k})\right]\right\} \frac{\bar{\tau}_{e}}{m_{e}^{*}} d^{3} k \\
L_{22}= & \frac{k_{B} T_{e}(\vec{r})}{4 \pi^{3}} \int_{B Z}\left\{f_{e}^{C}(\vec{r}, \vec{k}) \ln f_{e}^{C}(\vec{r}, \vec{k})+\left[1-f_{e}^{C}(\vec{r}, \vec{k})\right] \ln \left[1-f_{e}^{C}(\vec{r}, \vec{k})\right]\right\} \frac{\bar{\tau}_{e}}{m_{e}^{*}} d^{3} k \\
& \cdot \frac{k_{B}}{4 \pi^{3} n} \int_{B Z}\left\{f_{e}^{C}(\vec{r}, \vec{k}) \ln f_{e}^{C}(\vec{r}, \vec{k})+\left[1-f_{e}^{C}(\vec{r}, \vec{k})\right] \ln \left[1-f_{e}^{C}(\vec{r}, \vec{k})\right]\right\} d^{3} k .
\end{aligned}
$$


In Equation (23), applied for holes, the suitable quantity (see Equation (74)) is recognized as:

$$
\begin{aligned}
X_{1}= & \frac{\nabla \Phi_{p}(\vec{r})}{T_{h}(\vec{r})}, \quad X_{2}=\frac{\nabla T_{h}(\vec{r})}{T_{h}(\vec{r})} \\
L_{11}= & \frac{T_{h}(\vec{r})}{4 \pi^{3}} \int_{B Z}\left(1-f_{e}^{V}(\vec{r}, \vec{k})\right) \frac{\bar{\tau}_{h}}{m_{h}^{*}} d^{3} k \\
L_{12}= & L_{21}=\frac{k_{B} T_{h}(\vec{r})}{4 \pi^{3}} \int_{B Z}\left\{f_{e}^{v}(\vec{r}, \vec{k}) \ln f_{e}^{V}(\vec{r}, \vec{k})+\left[1-f_{e}^{V}(\vec{r}, \vec{k})\right] \ln \left[1-f_{e}^{V}(\vec{r}, \vec{k})\right]\right\} \frac{\bar{\tau}_{h}}{m_{h}^{*}} d^{3} k \\
L_{22}= & \frac{k_{B} T_{h}(\vec{r})}{4 \pi^{3}} \int_{B Z}\left\{f_{e}^{V}(\vec{r}, \vec{k}) \ln f_{e}^{V}(\vec{r}, \vec{k})+\left[1-f_{e}^{V}(\vec{r}, \vec{k})\right] \ln \left[1-f_{e}^{V}(\vec{r}, \vec{k})\right]\right\} \frac{\bar{\tau}_{h}}{m_{h}^{*}} d^{3} k \\
& \cdot \frac{k_{B}}{4 \pi^{3} p} \int_{B Z}\left\{f_{e}^{V}(\vec{r}, \vec{k}) \ln f_{e}^{V}(\vec{r}, \vec{k})+\left[1-f_{e}^{V}(\vec{r}, \vec{k})\right] \ln \left[1-f_{e}^{V}(\vec{r}, \vec{k})\right]\right\} d^{3} k
\end{aligned}
$$

\section{Postulate of the Variational Principle for the Semiconductor Heterostructure in Steady-State Conditions}

As was shown in Section 2, functional Equation (8) is not sufficient to obtain the proper expression for a non-equilibrium distribution function for electrons in CB and VB. To achieve this goal, we postulate applying a new variational principle obtained through a connection of functional Equation (8) with Gyarmati's principle expressed by relation Equation (23). In order to join them, we form the weighted potential $\Psi^{G}$ by multiplying equation Equation (23) by the mean relaxation time $\bar{\tau}_{e}$. This proposed principle (for $\mathrm{CB}$ electrons) reads as:

$$
\int_{V} \lambda_{e} d V=\int_{V}\left(m_{e}+\bar{\tau}_{e}\left[\sigma_{S_{e}}-\Psi\right]\right) d V=\max .
$$

Why should the acceptance $\bar{\tau}_{e}$ as the multiplier be a good choice? First, Gyarmati's principle remains valid if it is multiplied by any non-negative state function and the average relaxation time is a non-negative state function. Second, it is a characteristic time during which electron scattering takes place by collisions. This time determines band electron mobility and their drift velocity. We show further that the postulate will allow us to determine a non-equilibrium distribution function, which for small gradients of intensive parameters is identical to the function resulting from the BKEsolution, which will confirm the postulated validity.

By comparing the coefficient expression Equations (27)-(29), we note that the coefficient $L_{22}$ is orders of magnitude less than the other three coefficients. Thus, in equation Equation (26), we can skip the last component. Now, using Equation (68) and using relations Equations (9), (25)-(29), we can present the function $\lambda_{e}$ in the form of: 


$$
\begin{aligned}
\lambda_{e}= & \frac{1}{4 \pi^{3}} \int_{B Z} f_{e}^{C}(\vec{r}, \vec{k})\left(\chi(\vec{r})+e \Psi(\vec{r})-\varepsilon_{C}^{k}(\vec{k})+\Phi_{n}(\vec{r})\right) \frac{1}{T_{e}(\vec{r})} d^{3} k \\
& -\frac{1}{4 \pi^{3}} \frac{\nabla \Phi_{n}(\vec{r})}{T_{e}(\vec{r})} \int_{B Z} f_{e}^{C}(\vec{r}, \vec{k}) \bar{\tau} \frac{\hbar \vec{k}}{m_{e}^{*}} d^{3} k \\
& +\frac{k_{B}}{4 \pi^{3}} \int_{B Z}\left\{f_{e}^{C}(\vec{r}, \vec{k}) \ln f_{e}^{C}(\vec{r}, \vec{k})+\left[1-f_{e}^{C}(\vec{r}, \vec{k})\right] \ln \left[1-f_{e}^{C}(\vec{r}, \vec{k})\right]\right\} d^{3} k \\
& +\frac{k_{B}}{4 \pi^{3}} \frac{\nabla T_{e}(\vec{r})}{T_{e}(\vec{r})} \int_{B Z}\left\{f_{e}^{C}(\vec{r}, \vec{k}) \ln f_{e}^{C}(\vec{r}, \vec{k})+\left[1-f_{e}^{C}(\vec{r}, \vec{k})\right] \ln \left[1-f_{e}^{C}(\vec{r}, \vec{k})\right]\right\} \bar{\tau}_{e} \frac{\hbar \vec{k}}{m_{e}^{*}} d^{3} k \\
& +\frac{k_{B} T_{e}(\vec{r})}{4 \pi^{3}}\left(\frac{\nabla \Phi_{n}(\vec{r})}{T_{e}(\vec{r})}\right)\left(\frac{\nabla T_{e}(\vec{r})}{T_{e}(\vec{r})}\right) \int_{B Z}\left\{f_{e}^{C}(\vec{r}, \vec{k}) \ln f_{e}^{C}(\vec{r}, \vec{k})\right. \\
& -\frac{1}{2} \frac{T_{e}(\vec{r})}{4 \pi^{3}}\left(\frac{\nabla \Phi_{n}}{T_{e}}\right)^{2} \int_{B Z} f_{e}^{C}(\vec{r}, \vec{k}) \frac{\left(\bar{\tau}_{e}\right)^{2}}{m_{e}^{*}} d^{3} k .
\end{aligned}
$$

Equation (34) for holes takes the form of:

$$
\int_{V} \lambda_{h} d V=\int_{V}\left(m_{h}+\bar{\tau}_{h}\left[\sigma_{S_{h}}-\Psi\right]\right) d V=\max .
$$

Proceeding as with electrons, $\lambda_{h}$ will take the form of:

$$
\begin{aligned}
\lambda_{h} & =\frac{1}{4 \pi^{3}} \int_{B Z}\left[1-f_{e}^{V}(\vec{r}, \vec{k})\right]\left(-\chi(\vec{r})-e \Psi(\vec{r})-E_{g}(\vec{r})-\varepsilon_{V}^{k}(\vec{k})+\Phi_{p}(\vec{r})\right) \frac{1}{T_{h}(\vec{r})} d^{3} k \\
& +\frac{k_{B}}{4 \pi^{3}} \int_{B Z}\left\{f_{e}^{V}(\vec{r}, \vec{k}) \ln f_{e}^{V}(\vec{r}, \vec{k})+\left[1-f_{e}^{V}(\vec{r}, \vec{k})\right] \ln \left[1-f_{e}^{V}(\vec{r}, \vec{k})\right]\right\} d^{3} k \\
& +\frac{k_{B}}{4 \pi^{3}} \frac{\nabla T_{h}(\vec{r})}{T_{h}(\vec{r})} \int_{B Z}\left\{f_{e}^{V}(\vec{r}, \vec{k}) \ln f_{e}^{V}(\vec{r}, \vec{k})+\left[1-f_{e}^{V}(\vec{r}, \vec{k})\right] \ln \left[1-f_{e}^{V}(\vec{r}, \vec{k})\right]\right\} \bar{\tau}_{h} \frac{\hbar \vec{k}}{m_{h}^{*}} d^{3} k \\
& -\frac{k_{B} T_{h}(\vec{r})}{4 \pi^{3}}\left(\frac{\nabla \Phi_{p}(\vec{r})}{T_{h}(\vec{r})}\right)\left(\frac{\nabla T_{h}(\vec{r})}{T_{h}(\vec{r})}\right) \int_{B Z}\left\{f_{e}^{V}(\vec{r}, \vec{k}) \ln f_{e}^{V}(\vec{r}, \vec{k})\right. \\
& \left.\quad+\left[1-f_{e}^{V}(\vec{r}, \vec{k})\right] \ln \left[1-f_{e}^{V}(\vec{r}, \vec{k})\right]\right\} \frac{\left(\bar{\tau}_{h}\right)^{2}}{m_{h}^{*}} d^{3} k \\
& +\frac{1}{4 \pi^{3}} \frac{\nabla \Phi_{h}(\vec{r})}{T_{h}(\vec{r})} \int_{B Z}\left[1-f_{e}^{V}(\vec{r}, \vec{k})\right] \bar{\tau}_{h} \frac{\hbar \vec{k}}{m_{h}^{*}} d^{3} k-\frac{1}{2} \frac{T_{h}(\vec{r})}{4 \pi^{3}}\left(\frac{\nabla \Phi_{p}(\vec{r})}{T_{h}(\vec{r})}\right)^{2} \int_{B Z} f_{e}^{V}(\vec{r}, \vec{k}) \frac{\left(\bar{\tau}_{h}\right)^{2}}{m_{h}^{*}} d^{3} k .
\end{aligned}
$$

\subsection{The Non-Equilibrium Distribution Function for Electrons}

Functional Equation (34) achieves maximum value if the function $\lambda_{e}$ meets the E-L equation, which means:

$$
\frac{\partial \lambda_{e}}{\partial f_{e}^{C}}=0 .
$$

The solution of Equation (39) is the following function:

$$
\begin{aligned}
& f_{e}^{C}(\vec{r}, \vec{k})= \\
& {\left[1+\exp \left(\frac{-\chi(\vec{r})-e \Psi(\vec{r})+\varepsilon_{C}^{k}(\vec{k})+\bar{\tau}_{e} \nabla \Phi_{n}(\vec{r}) \frac{\hbar \vec{k}}{m_{e}^{*}}+\frac{1}{2}\left(\nabla \Phi_{n}(\vec{r})\right)^{2}\left(\bar{\tau}_{e}\right)^{2} \frac{1}{m_{e}^{*}}-\Phi_{n}(\vec{r})}{k_{b}\left[T_{e}(\vec{r})+\left(\bar{\tau}_{e} \frac{\hbar \vec{k}}{m_{e}^{*}}+\nabla \Phi_{n}(\vec{r})\left(\bar{\tau}_{e}\right)^{2} \frac{1}{m_{e}^{*}}\right) \nabla T_{e}(\vec{r})\right]}\right)\right]^{-1}}
\end{aligned}
$$




\subsection{The Non-Equilibrium Distribution Function for Holes}

Functional Equation (37) achieves maximum value, if the E-L equation reads as:

$$
\frac{\partial \lambda_{h}}{\partial f_{e}^{V}}=0
$$

The solution of Equation (41) is the following function:

$$
\begin{aligned}
& f_{e}^{V}(\vec{r}, \vec{k})= \\
& {\left[1+\exp \left(\frac{-\chi(\vec{r})-e \Psi(\vec{r})-E_{g}(\vec{r})-\varepsilon_{V}^{k}(\vec{k})-\bar{\tau}_{h} \nabla \Phi_{p}(\vec{r}) \frac{\hbar \vec{k}}{m_{h}^{*}}-\frac{1}{2}\left(\nabla \Phi_{p}(\vec{r})\right)^{2}\left(\bar{\tau}_{h}\right)^{2} \frac{1}{m_{h}^{*}}+\Phi_{p}(\vec{r})}{k_{b}\left[T_{h}(\vec{r})+\left(\bar{\tau}_{h} \frac{\hbar \vec{k}}{m_{h}^{*}}-\nabla \Phi_{p}(\vec{r})\left(\bar{\tau}_{h}\right)^{2} \frac{1}{m_{h}^{*}}\right) \nabla T_{h}(\vec{r})\right]}\right)\right]^{-1}}
\end{aligned}
$$

While the distribution function of holes $f_{h}$ will take the form of:

$$
\begin{aligned}
& f_{h}(\vec{r}, \vec{k})=1-f_{e}^{V}(\vec{r}, \vec{k})= \\
& {\left[1+\exp \left(\frac{\chi(\vec{r})+e \Psi(\vec{r})+E_{g}(\vec{r})+\varepsilon_{V}^{k}(\vec{k})+\bar{\tau}_{h} \nabla \Phi_{p}(\vec{r}) \frac{\hbar \vec{k}}{m_{h}^{*}}+\frac{1}{2}\left(\nabla \Phi_{p}(\vec{r})\right)^{2}\left(\bar{\tau}_{h}\right)^{2} \frac{1}{m_{h}^{*}}-\Phi_{p}(\vec{r})}{k_{b}\left[T_{h}(\vec{r})+\left(\bar{\tau}_{h} \frac{\hbar \vec{k}}{m_{h}^{*}}-\nabla \Phi_{p}(\vec{r})\left(\bar{\tau}_{h}\right)^{2} \frac{1}{m_{h}^{*}}\right) \nabla T_{h}(\vec{r})\right]}\right)\right]^{-1}}
\end{aligned}
$$

\section{Verification of the Obtained Results}

Here, we verify the obtained relation Equations (40) and (43) by comparing them with suitable results obtained by other authors using another method of analysis. The agreement of the results would be the confirmation that the postulated variational principles (relations Equations (34) and (37)) are suitable for analyzing the steady-state conditions in semiconductor heterostructures. Let us begin our considerations for the function $f_{e}^{C}$. We compare expression Equation (40) with Expression (5.1) obtained by Marshak and van Vliet [2] by a solution of the KBE under the assumption that the values of $\nabla \Phi_{n}$ and $\nabla T_{e}$ are low. We decompose relation Equation (40) into the Taylor series around $\Phi_{n}$ and $T_{e}$ under the assumption that $\nabla \Phi_{n}$ and $\nabla T_{e}$ are of low values. As a result, we obtain the relation of:

$$
\begin{aligned}
f_{e}^{C} \approx & {\left[1+\exp \left(\frac{-\chi-e \Psi+\varepsilon_{C}^{k}(\vec{k})-\Phi_{n}}{k_{B} T_{e}}\right)\right]^{-1} } \\
& -\frac{\exp \left(\frac{-\chi-e \Psi+\varepsilon_{C}^{k}(\vec{k})-\Phi_{n}}{k_{B} T_{e}}\right)}{\left[1+\exp \left(\frac{-\chi-e \Psi+\varepsilon_{C}^{k}(\vec{k})-\Phi_{n}}{k_{B} T_{e}}\right)\right]^{2}}\left(\frac{\bar{\tau}_{e}}{k_{B} T_{e}} \nabla \Phi_{n} \frac{\hbar \vec{k}}{m_{e}^{*}}-\frac{\left(-\chi-e \Psi+\varepsilon_{C}^{k}(\vec{k})-\Phi_{n}\right)}{k_{B}\left(T_{e}\right)^{2}} \bar{\tau}_{e} \frac{\hbar \vec{k}}{m_{e}^{*}} \nabla T_{e}\right)
\end{aligned}
$$

It is easy to notice that expression Equation (44) is the same as Expression (5.1) in the paper of Marshak and van Vliet [2]. 
Similarly, by decomposing the function $f_{h}$ around $\Phi_{p}$ and $T_{h}$ under the assumption that $\nabla \Phi_{p}$ and $\nabla T_{h}$ are of small values and by confining ourselves to the first order words, we are going to obtain the following:

$$
\begin{aligned}
f_{h} \approx & {\left[1+\exp \left(\frac{\chi+e \Psi+E_{g}+\varepsilon_{V}^{k}(\vec{k})-\Phi_{p}}{k_{B} T_{h}}\right)\right]^{-1} } \\
& -\frac{\exp \left(\frac{\chi+e \Psi+E_{g} \varepsilon_{V}^{k}(\vec{k})-\Phi_{p}}{k_{B} T_{h}}\right)}{\left[1+\exp \left(\frac{\chi+e \Psi+E_{g} \varepsilon_{V}^{k}(\vec{k})-\Phi_{p}}{k_{B} T_{h}}\right)\right]^{2}}\left(\frac{\bar{\tau}_{h}}{k_{B} T_{h}} \nabla \Phi_{p} \frac{\hbar \vec{k}}{m_{h}^{*}}-\frac{\chi+e \Psi+E_{g}+\varepsilon_{V}^{k}(\vec{k})-\Phi_{p}}{k_{B}\left(T_{h}\right)^{2}} \frac{\hbar \vec{k}}{m_{h}^{*}} \nabla T_{h}\right)
\end{aligned}
$$

Expression Equation (45) can be obtained similarly as what was done by Marshak and van Vliet [2] by solving the BKE under the assumption that the gradients $\nabla \Phi_{p}$ and $\nabla T_{h}$ are low. Thus, expression Equations (44) and (45) obtained in this paper are in agreement with the results obtained by solving the BKE in the special case. This confirms that the forms of functionals Equations (34) and (37) are proper and can be used to analyze semiconductor heterostructures in steady-state conditions

Expression Equations (40) and (43) are more general than the expressions for non-equilibrium distribution functions, obtained by solving the BKE. What is more, non-equilibrium distribution functions have the form of the Fermi-Dirac distribution function (with four additional components). The physical interpretation of these components is relatively simple. We explain it for $f_{e}^{C}$. The physical interpretation of suitable components in $f_{h}$ is analogous to those in $f_{e}^{C}$.

The expression $\left(\frac{\hbar \vec{k}}{m_{e}^{*}} \bar{\tau}_{e}+\nabla \Phi_{n} \frac{\left(\bar{\tau}_{e}\right)^{2}}{m_{e}^{*}}\right) \cdot \nabla T_{e}$ is equal to $d T_{e}$, the change of the electron's temperature in the time period equal to the mean relaxation time $\bar{\tau}_{e}$ (Figure 2).

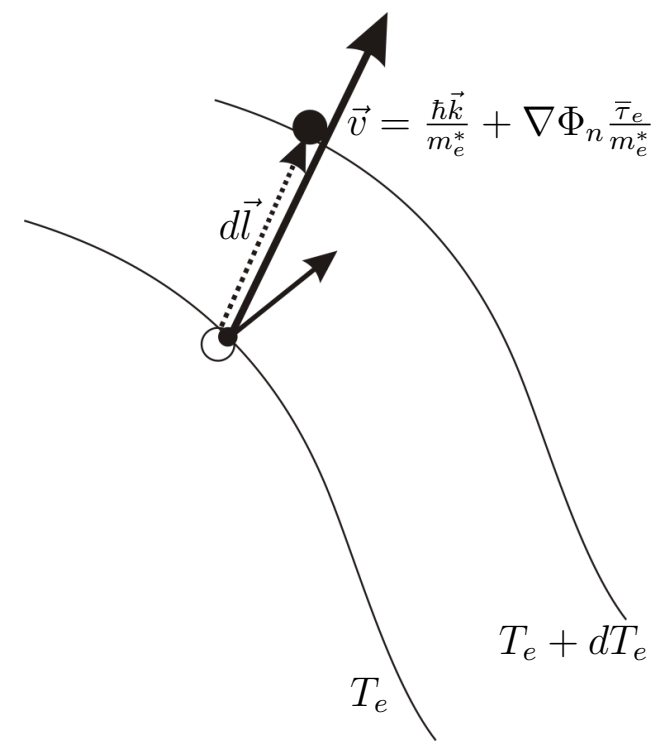

Figure 2. An electron having thermal velocity $\frac{\hbar \vec{k}}{m_{e}^{*}}+\nabla \Phi_{n} \frac{\bar{\tau}_{e}}{m_{e}^{*}}$ (total electron velocity with subtracted drift velocity caused by $\nabla \Phi_{n}$ ) at the point on the isotherm with temperature $T$ in the time period equaling the mean relaxation time $\bar{\tau}_{e}$ moves to the point on the isotherm with temperature $T_{e}+d T_{e}$. Effective growth of electron temperature is $d T_{e}=d \vec{l} \cdot \nabla T_{e}=$ $\left(\frac{\hbar \vec{k}}{m_{e}^{*}} \bar{\tau}_{e}+\nabla \Phi_{n} \frac{\left(\bar{\tau}_{e}\right)^{2}}{m_{e}^{*}}\right) \cdot \nabla T_{e} \cdot d \vec{l}$ is the displacement vector of electron in time $\bar{\tau}_{e}$. 
The expression $\bar{\tau}_{e}^{k} \frac{\hbar \vec{k} \cdot \nabla \Phi_{n}(\vec{r})}{m_{e}^{*}(\vec{r})}$ is recognized (Figure 3) as a change of electron kinetic energy caused by the movement of the electron in the region where the gradient of quasi-Fermi energy occurs. The sign of this change depends on the direction of the electron velocity. The expression $\frac{1}{2}\left(\nabla \Phi_{n}\right)^{2} \frac{\left(\bar{\tau}_{e}\right)^{2}}{m_{e}^{*}}$ is found as an increase of electron energy caused by the force $-\nabla \Phi_{n}$ working on the distance $\frac{1}{2} a\left(\bar{\tau}_{e}\right)^{2}=-\frac{1}{2} \frac{\nabla \Phi_{n}}{m_{e}^{*}}\left(\bar{\tau}_{e}\right)^{2}$.

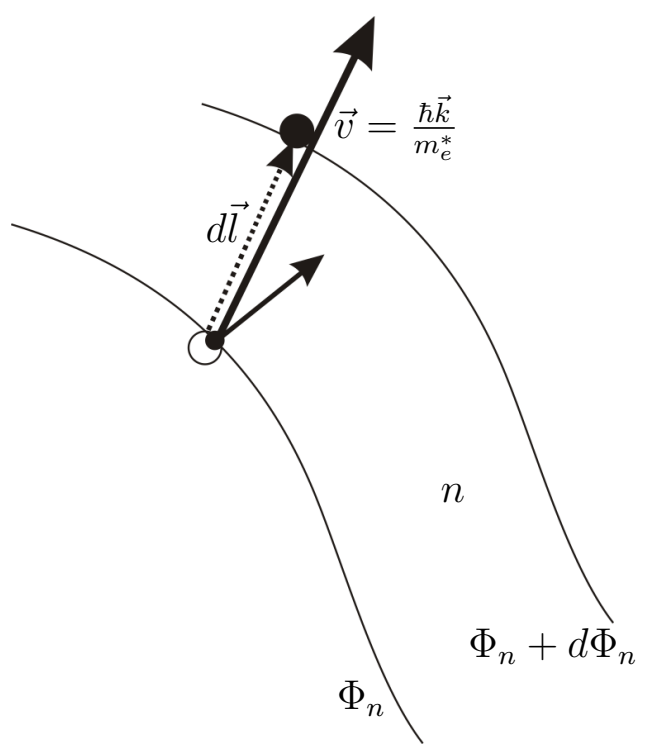

Figure 3. The electron having velocity $\vec{v}=\frac{\hbar \vec{k}}{m_{e}^{*}}$ moves from the position where quasi-Fermi energy equals $\Phi_{n}$ in the time period equaling $\bar{\tau}_{e}$ to the point where quasi-Fermi energy equals $\Phi_{n}+d \Phi_{n}$, where $d \Phi_{n}=d \vec{l} \cdot \nabla \Phi_{n}$. The displacement vector $d \vec{l}$ is equal to $d \vec{l}=\frac{\hbar \vec{k}}{m_{e}^{*}} \bar{\tau}_{e}$. This leads to a change of the electron kinetic energy $\bar{\tau}_{e}^{k \frac{\hbar \vec{k} \cdot \nabla \Phi_{n}(\vec{r})}{m_{e}^{*}(\vec{r})}}=d \varepsilon_{C}^{k}$.

\section{The Calculation Method and Its Exemplary Results}

We discuss here the calculation method consisting of calculating the electron and hole distribution function, as well as presenting the exemplary results of the calculations for electrons (relation Equation (40)). The calculations will involve ternary semiconductor compounds of mercury cadmium telluride (MCT) $\left(\mathrm{Cd}_{x} \mathrm{Hg}_{1-x} \mathrm{Te}\right)$ with two chosen values of $x$, a mole fraction of CdTe $(x=0.165$ and $x=0.3$ ), a concentration of donors $N_{D}=10^{15} \mathrm{~cm}^{-3}$ and acceptors $N_{A}=10^{13} \mathrm{~cm}^{-3}$. Data on the physical parameters of the material can be found, for example, in the book edited by Capper [26]. While conducting the numerical modeling of devices, we are not able to calculate non-equilibrium distribution functions at the heterostructure selected points directly using only relation Equations (40) and (43). The calculations must be conducted by iterative methods, because Equations (40) and (43) are highly non-linear. In turn, these values are dependent on external forces maintaining a steady state and on a distribution function. First, our calculations begin for a thermal equilibrium state by solving the Poisson equation with iterative methods for given spatial distributions of a molar composition and doping. This allows the determination of the spatial distribution of electric potential, as well as the electron, holes and ionized dopant concentrations. In order to determine these concentrations, the Fermi-Dirac functions applicable to the thermal equilibrium state are used. The accurate description of this method is in the paper of Jóźwikowska [27]. 
Next, by changing the boundary conditions on electrical contacts due to the value of the applied voltage, we solve the system of four or six transport equations with iterative methods. We gradually increase the voltage until it reaches a desired value. Subsequent changes to the voltage value are the condition for the convergence and stability of the iterative method used. In the first iterative step for the initial voltage, we make calculations by using the non-equilibrium distribution functions in the form of the Fermi-Dirac function for thermal equilibrium. In these functions, the Fermi level is replaced with quasi-Fermi levels, and we take into account that the electron and holes own a thermodynamic temperature, which can be different from the lattice temperature. For electrons in the conduction band and valence band, those are Equations (13) and (14), respectively. In subsequent iterations, we already use distribution functions in the form of Equations (40) and (43). Using these, we calculate both the carrier concentration, as well as the average relaxation time defined by Equation (71). Counting the relaxation time in $\mathrm{MCT}$, we take into account two mechanisms of scattering: ionized impurity scattering and polarized optical phonon scattering [13]. Due to the fact that the effective mass of electrons in a $\mathrm{HgCdTe}$ narrow gap is more than an order of magnitude less than the effective mass of holes, effects related to the gradient influence of intensive parameters are in the case of the electron distribution function much stronger than for holes. Therefore, the exemplary calculation results are shown for the non-equilibrium distribution function for electrons, as they are the most spectacular. As we analyzed the semiconductor instruments, temperature gradients are usually very small, and their impact on the non-equilibrium distribution function is incomparably smaller than the impact of the quasi-Fermi level gradients. In the cases presented here, the influence of a temperature gradient on the function $f_{e}^{C}$ is negligible.

Figure $4 \mathrm{a}-4 \mathrm{f}$ presents the dependence of the function on the angle, which the wave vector $\vec{k}$ creates with the vector $\nabla \Phi_{n}$ for selected modules of the vector $\vec{k}$ and the selected gradients $\nabla \Phi_{n}$ (see Figure 5). Figure $4 \mathrm{a}-4 \mathrm{c}$ relates to the structure of the molar composition $x=0.165$ and the temperature $T=300 \mathrm{~K}$, and Figure $4 \mathrm{~d}-4 \mathrm{f}$ relates to the structure with parameters $x=0.3$ and $T=300 \mathrm{~K}$.

Now, it is evident that distribution functions determined by relation Equation (40) are not even in the wave vector, in contrast to those determined by relation Equation (13). $\bar{\tau}_{e} \nabla \Phi_{n} \frac{\hbar \vec{k}}{m_{e}^{*}}$ determines it. When the dot product $\nabla \Phi_{n} \cdot \vec{k}$ is positive, it increases the potential energy of a moving electron at the expense of kinetic energy. Reducing the kinetic energy leads to increasing the value of $f_{e}^{C}$. When the movement direction is the opposite, then the electron potential energy decreases, and its kinetic energy increases. Hence, the increase in value of the function $f_{e}^{C}$ is with the increase at the angle $\alpha$. The impact of the component $\bar{\tau}_{e} \nabla \Phi_{n} \frac{\hbar \vec{k}}{m_{e}^{*}}$ on the function $f_{e}^{C}$ becomes increased with both the increase of module $\vec{k}$ vector and with the gradient value $\nabla \Phi_{n}$, which is well illustrated by Figure 4. 


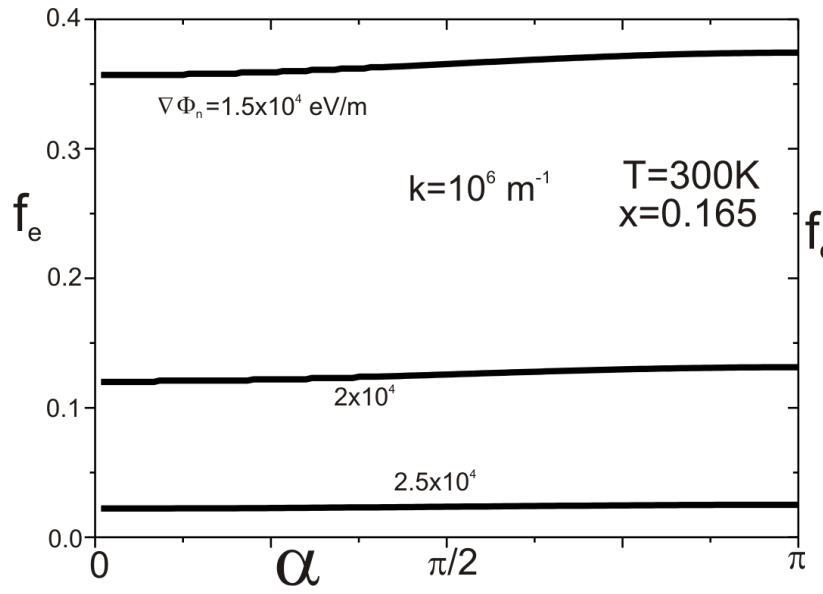

(a)

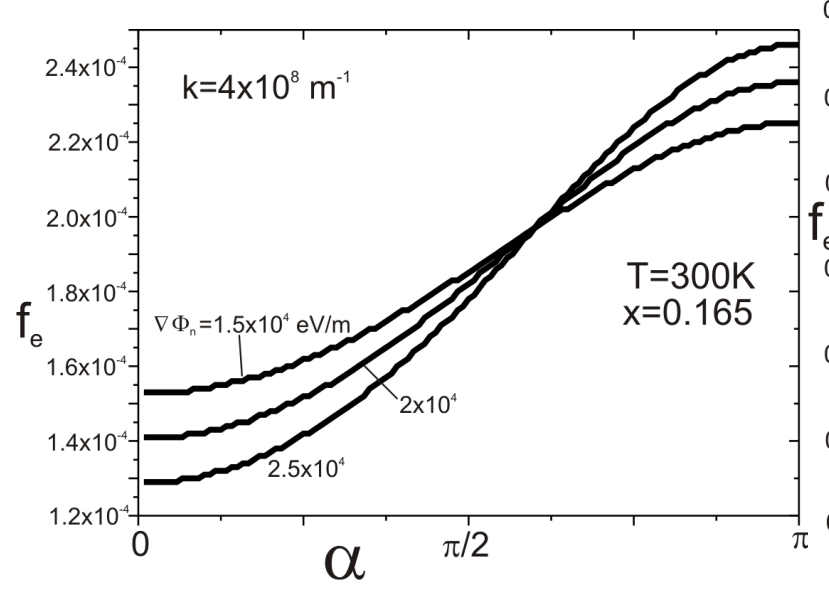

(c)

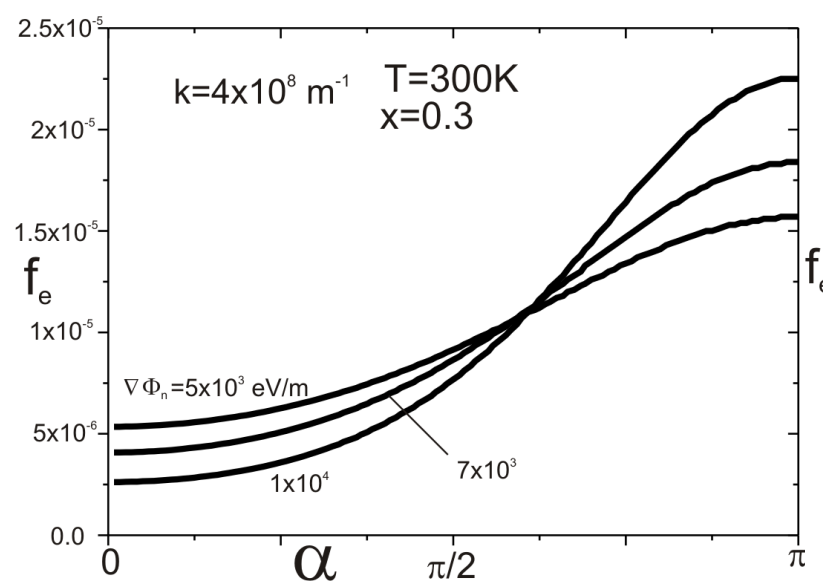

(e)

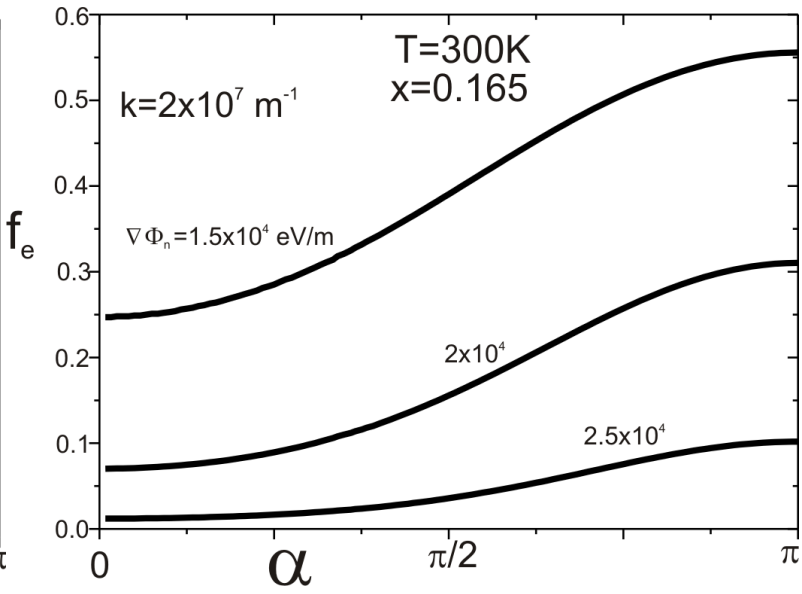

(b)

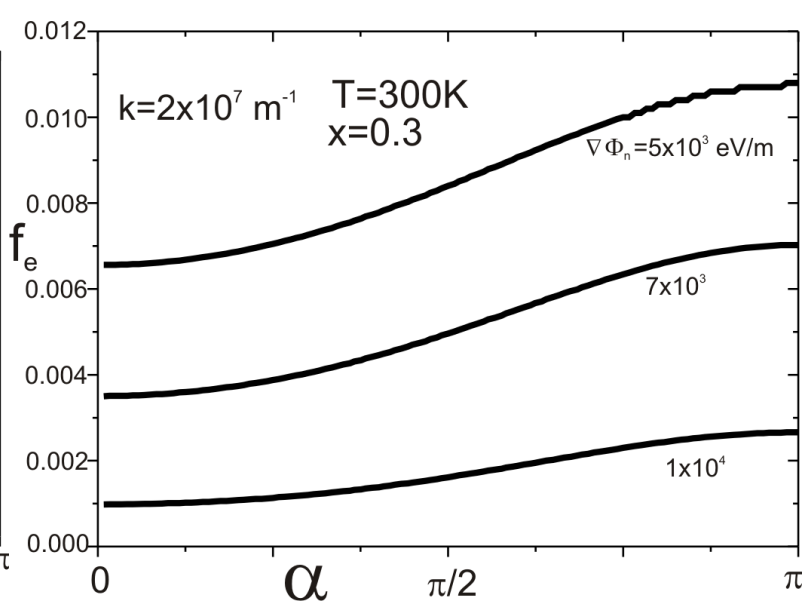

(d)

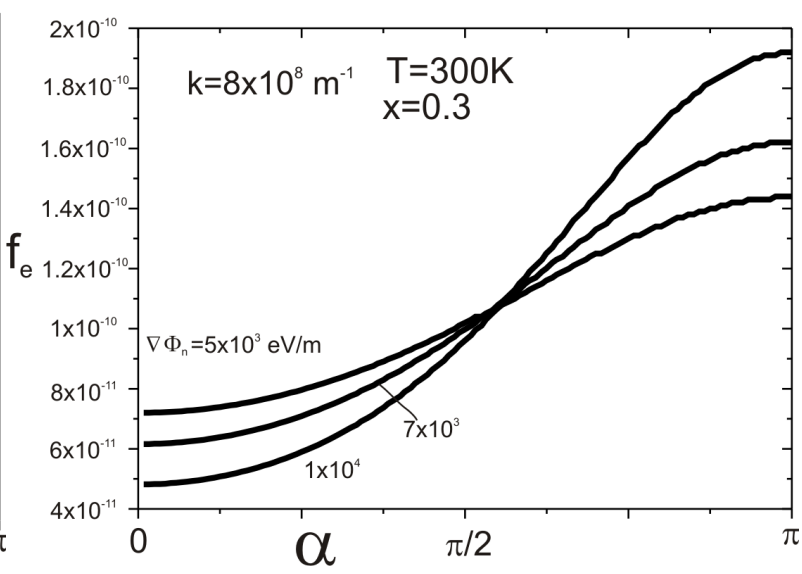

(f)

Figure 4. Dependence $f_{e}$ on the angle $\alpha$ that the wave vector $\vec{k}$ creates with the vector $\nabla \Phi_{n}$ for the selected modules of the vector $\vec{k}$ and the selected gradients $\nabla \Phi_{n}$ (numbers with curves). (a-c) The structure of $x=0.165, T=300 \mathrm{~K}$; and $(\mathbf{d}-\mathbf{f})$ the structure of $x=0.3$, $T=300 \mathrm{~K}$. 


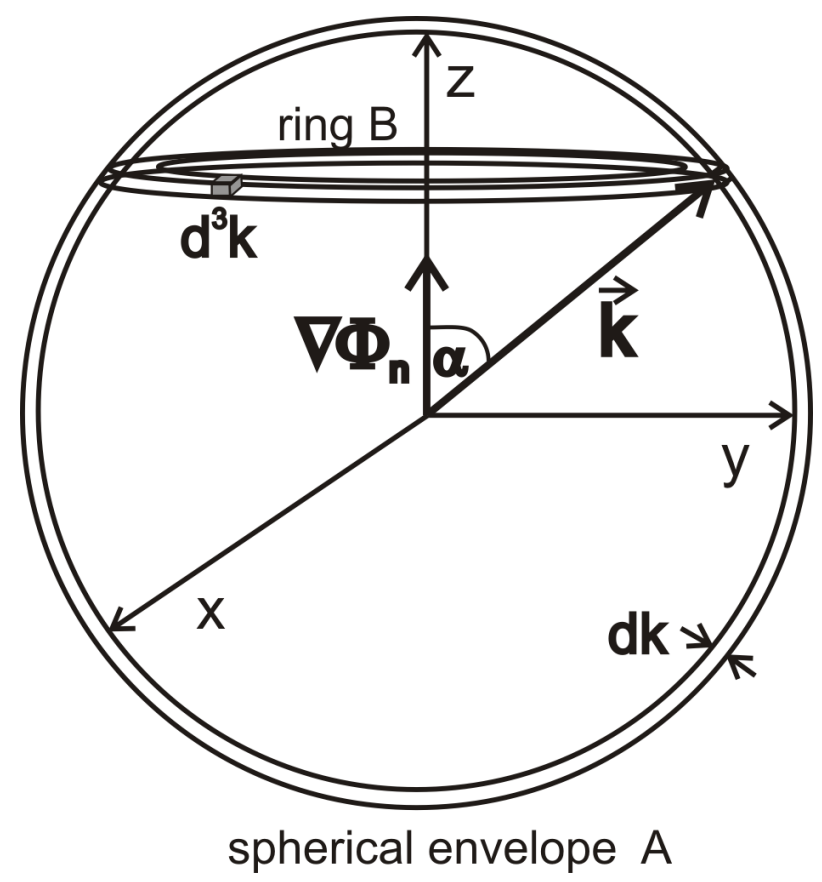

Figure 5. In the space of the wave vector $\vec{k}$, we distinguish the $z$ direction parallel to the vector $\nabla \Phi_{n}$. This space is divided into chase-space cells having a volume of $d^{3} k=4 \pi^{3} / d V$. Here, $V$ denotes the volume of the semiconductor. The probability that any cell is occupied by an electron is determined by the distribution function $f_{e}^{C}$ (relation Equation (40)) and depends on the absolute value of the electron wave vector $\vec{k}$ and the angle $\alpha$ between vector $\vec{k}$ and the $z$ direction. For all phase space cells in Ring B, the values of $f_{e}^{C}$ are the same.

Figure 6 presents the dependence of $f_{e}^{C}$ on the wave vector module $\vec{k}$ for the selected gradient values $\nabla \Phi_{n}$ (numbers with curves). The presented values of the function $f_{e}^{C}$ are mean values for cells that form the spherical Envelope A (see Figure 5). Figure 6a describes the structure with the parameters of $x=0.165, T=300 \mathrm{~K}$; Figure $6 \mathrm{~b}, x=0.3, T=300 \mathrm{~K}$; and Figure $6 \mathrm{c}, x=0.165, T=80 \mathrm{~K}$. For $k<10^{7} \mathrm{~m}^{-1}, f_{e}^{C}$ weakly depends on $k$, but rapidly disappears with increasing $k$ for $k>10^{8} \mathrm{~m}^{-1}$. The reason for this failure is the kinetic energy $\varepsilon_{C}^{k}(\vec{k})$ increase, which is a quadratic function of the wave vector $\vec{k}$. Increasing the kinetic energy component $\frac{1}{2}\left(\nabla \Phi_{n}\right)^{2} \frac{\left(\bar{\tau}_{e}\right)^{2}}{m_{e}^{*}}$ is the reason for the observed decrease in the value of $f_{e}^{C}$ with the increasing values of $\nabla \Phi_{n}$. However, the increase of the value of $\nabla \Phi_{n}$ increases the role of the component $\bar{\tau}_{e} \nabla \Phi_{n} \frac{\hbar \vec{k}}{m_{e}^{*}}$, which depending on the sign of the scalar product $\nabla \Phi_{n} \cdot \vec{k}$, can cause either an increase or decrease in the value of $f_{e}^{C}$. This component of the overall balance will increase the average value of the function $f_{e}^{C}$, which is noticeable for sufficiently large values of the gradient $\nabla \Phi_{n}$. This is particularly shown in Figure 6c, where the influence of this component in the range of values $10^{7} \mathrm{~m}^{-1}<k<10^{8} \mathrm{~m}^{-1}$ seems to be dominant. This is achieved by the addition of average relaxation time, which grows with a decreasing temperature. Calculated by us, $\bar{\tau}_{e}$, for the material $x=0.165$ at $T=300 \mathrm{~K}$ is $4.17 \times 10^{-12} \mathrm{~s}$ and at $T=80 \mathrm{~K}$ is $7.3 \times 10^{-12} \mathrm{~s}$. 


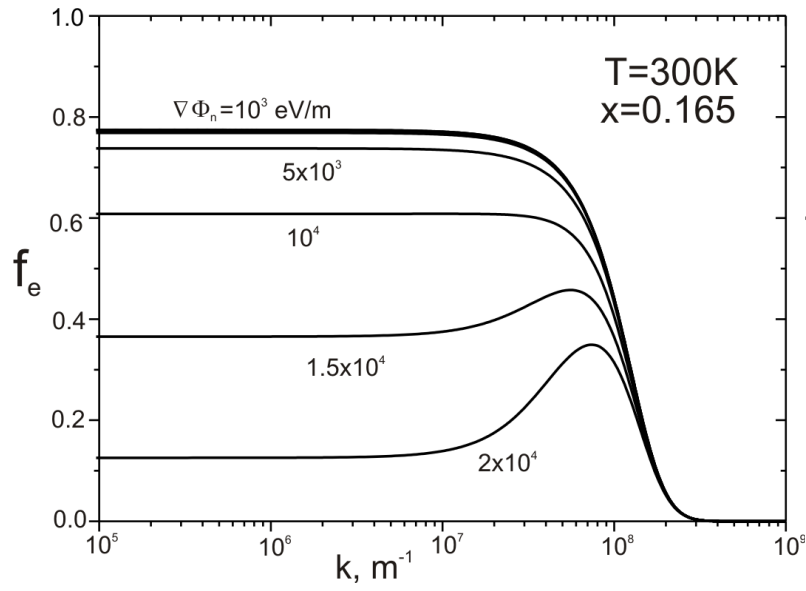

(a)

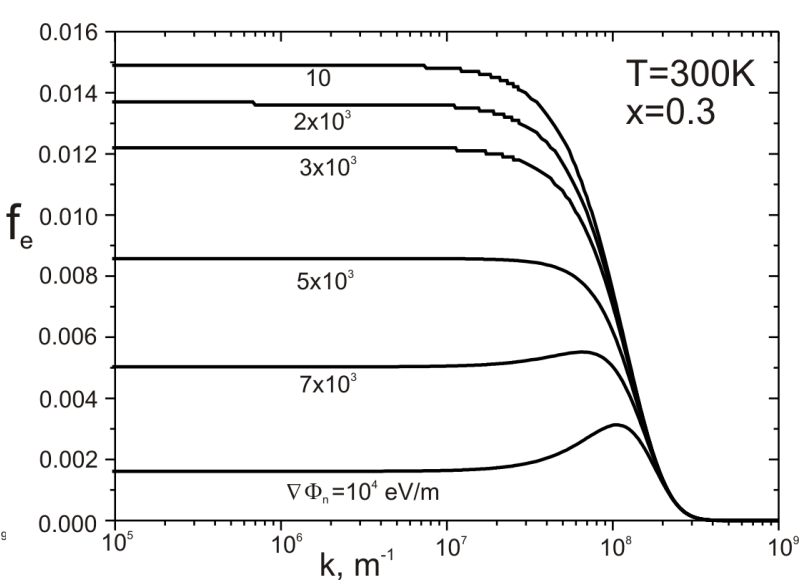

(b)

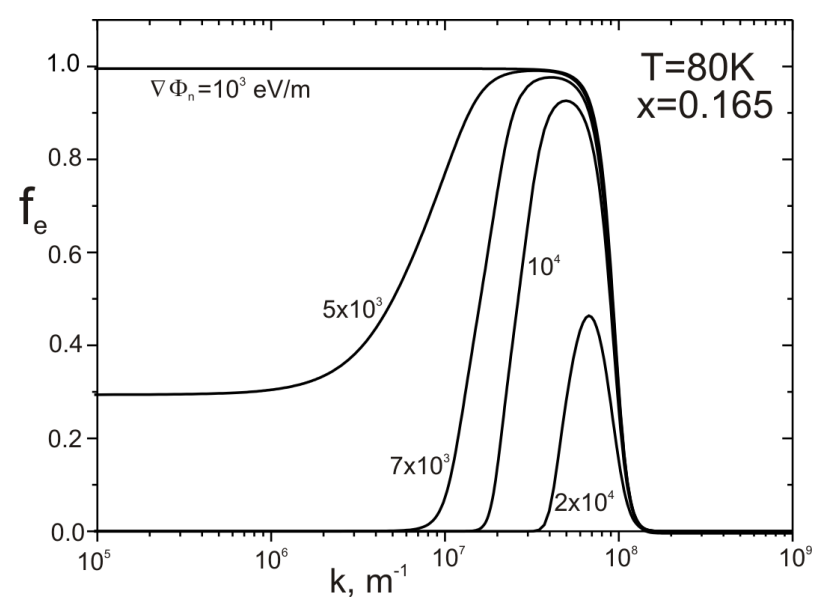

(c)

Figure 6. Dependence of the average $f_{e}$ on $k$, the module of the wave vector $\vec{k}$, for the selected gradients $\nabla \Phi_{n}$ (numbers with curves). (a) The structure of $x=0.165, T=300 \mathrm{~K}$; (b) $x=0.3, T=300 \mathrm{~K}$; (c) $x=0.165, T=80 \mathrm{~K}$. The figures present the mean value of the function distribution of phase cells located in the spherical Envelope A (see Figure 5).

Figure 7a-7c presents the dependence of electron concentration on the value of $\nabla \Phi_{n}$. Figure 7a shows the structure with parameters $x=0.165, T=300 \mathrm{~K}$; Figure $7 \mathrm{~b}, x=0.3, T=300 \mathrm{~K}$; and Figure $7 \mathrm{c}, x=0.165, T=80 \mathrm{~K}$. In all three cases, the electron concentration remains almost constant for gradients $\nabla \Phi_{n}<10^{4} \mathrm{eV} \mathrm{m}^{-1}$. The increase of the gradient $\nabla \Phi_{n}$ up to the value of $10^{5} \mathrm{eV} \mathrm{m}^{-1}$ results in a reduction of the concentration in all cases by several orders of magnitude. The obtained values of electron concentration are in good agreement with the experimental data presented by Capper [26]. 


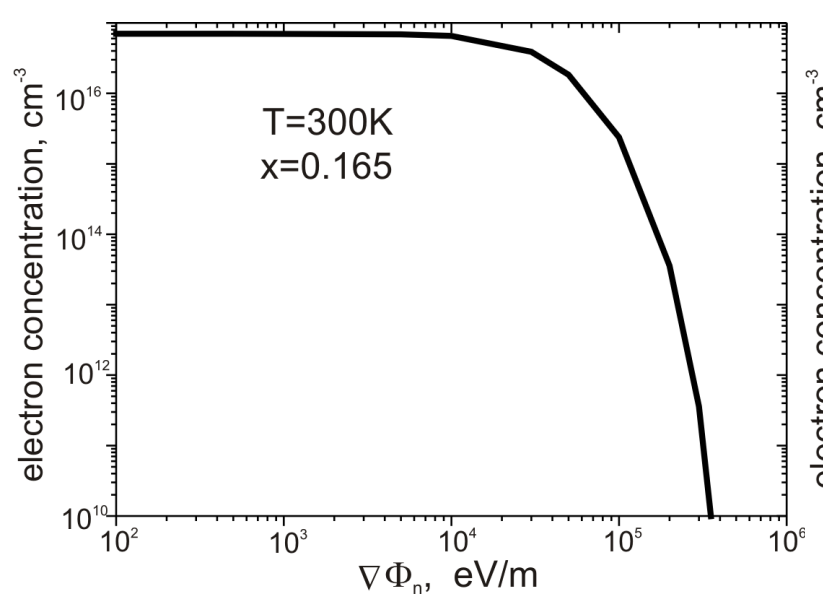

(a)

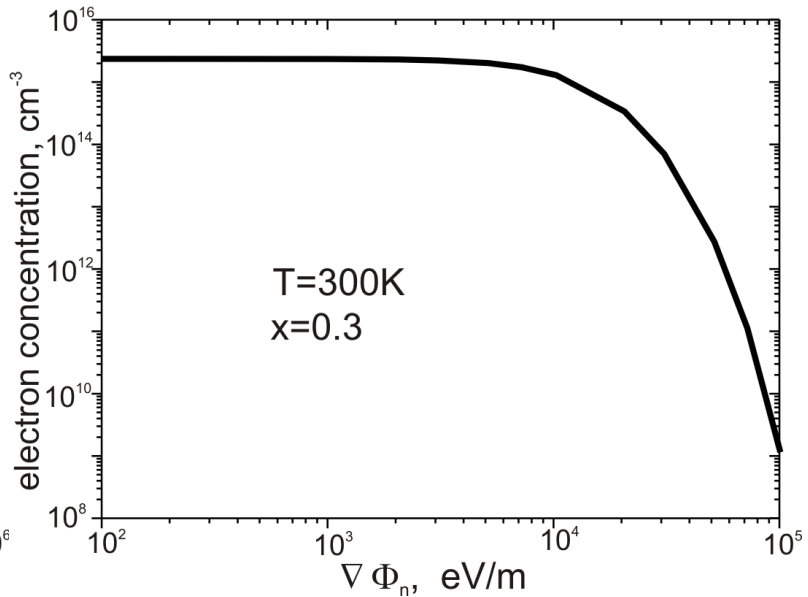

(b)

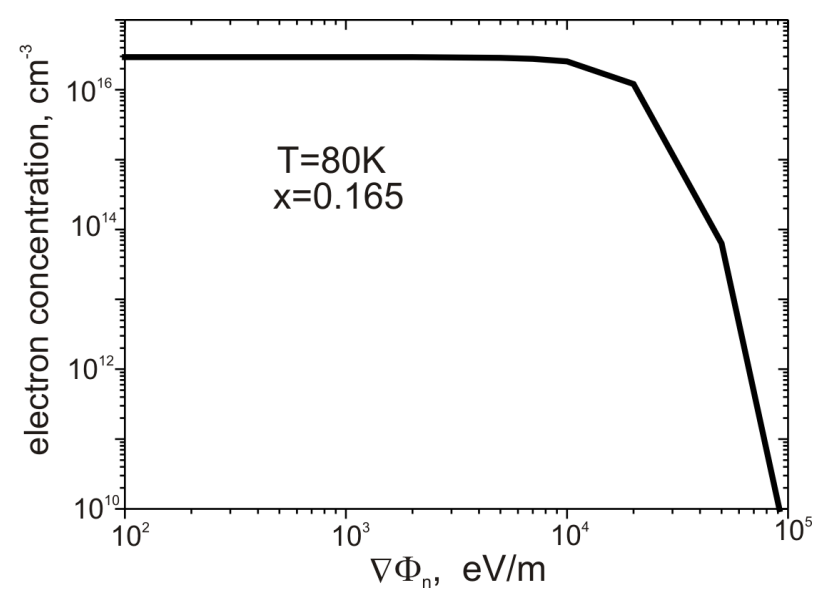

(c)

Figure 7. Electron concentration in the function for the structures of: (a) $x=0.165, T=$ $300 \mathrm{~K}$; (b) $x=0.3, T=300 \mathrm{~K}$; and (c) $x=0.165, T=80 \mathrm{~K}$.

\section{Conclusions}

Based on the postulates of local equilibrium, as well as on the integral form of Gyarmati's variational principle in the force representation, we have derived distribution functions for an electron and holes. Thus, it is an alternative method commonly solving the BKE with the relaxation times' approximation used. The obtained expressions have the form of the Fermi-Dirac distribution function with four additional components. We have shown the physical interpretation of these components as a change of effective carrier temperature and kinetic energy in the regions where gradients of temperature and electrochemical potential occur. These expressions should be used especially in areas where there are heavy electro-chemical potential gradients, such as the p-n junction, heterojunction, metal-semiconductor connectors, etc. If one assumes that these gradients are weak, our results are in excellent agreement with previous expressions obtained by solving the BKE. The presented method should be developed to additionally consider many galvanomagnetic and thermomagnetic effects, and so on, which may be important in some applications. 


\section{Acknowledgments}

The work has been undertaken under the financial support of the Polish National Science Centre as research Project No. DEC-2013/08/M/ST7/00913 and Project No. POIG.01.03.01-14-016/08 "New Photonic Materials and Their Advanced Application".

\section{Author Contributions}

The theoretical idea is attributed to K.J. and A.J. M.N. is responsible for the numerical calculations. Both authors have read and approved the final manuscript.

\section{Conflicts of Interest}

The authors declare no conflict of interest.

\section{Appendix}

\section{A. The Energy and Entropy of Electrons and Holes in a Semiconductor Heterostructure}

Figure 8 shows a two-band energy diagram for the general case of a semiconductor heterostructure. The concept of the local vacuum level $E_{i}$, which represents the energy of an electron at a given point if it is at rest and free from the influence of the crystal potential, was introduced by Marshak and van Vliet [2]. Thus, $E_{i}$ gives the spatial variation of electrostatic potential $\Psi$. The reference level $E_{0}$ may be an arbitrarily-chosen value of the energy. In our paper, we have assumed $E_{0}=0$ without any loss of generality. $E_{V}$ is the $\mathrm{VB}$ edge, and $E_{C}$ is the $\mathrm{CB}$ edge.

The energy of a single electron in $\mathrm{CB}\left(-\chi-e \Psi+\varepsilon_{C}^{k}\right)$ contains element $\varepsilon_{C}^{k}$, which denotes the electron kinetic energy. $\varepsilon_{C}^{k}$ is the function of wave vector $\vec{k}$. The density of CB electron energy $u_{e}$ in an element of volume determined by position vector $\vec{r}$ is expressed by:

$$
u_{e}(\vec{r})=\frac{1}{4 \pi^{3}} \int_{B Z} f_{e}^{C}(\vec{r}, \vec{k})\left(-\chi(\vec{r})-e \Psi(\vec{r})+\varepsilon_{C}^{k}(\vec{k})\right) d^{3} k
$$

Integration is carried out inside the Brillouin zone (BZ), and $f_{e}^{C}(\vec{r}, \vec{k})$ is the distribution function for CB electrons. The density of the hole energy, $u_{h}$, reads:

$$
u_{h}(\vec{r})=\frac{1}{4 \pi^{3}} \int_{B Z}\left(1-f_{e}^{V}(\vec{r}, \vec{k})\right)\left(\chi(\vec{r})+e \Psi(\vec{r})+E_{g}(\vec{r})+\varepsilon_{V}^{k}(\vec{k})\right) d^{3} k,
$$

where $\varepsilon_{V}^{k}(\vec{k})$ is the hole kinetic energy. $f_{e}^{V}(\vec{r}, \vec{k})$ is the distribution function for VB electrons. The density of the $\mathrm{CB}$ electron entropy reads [28]:

$$
s_{e}(\vec{r})=-k_{B} \frac{1}{4 \pi^{3}} \int_{B Z}\left\{f_{e}^{C}(\vec{r}, \vec{k}) \ln f_{e}^{C}(\vec{r}, \vec{k})+\left[1-f_{e}^{C}(\vec{r}, \vec{k})\right] \ln \left[1-f_{e}^{C}(\vec{r}, \vec{k})\right]\right\} d^{3} k .
$$

The density of the VB electron entropy equal to the density of the hole entropy is expressed by [28]:

$$
s_{h}(\vec{r})=-k_{B} \frac{1}{4 \pi^{3}} \int_{B Z}\left\{f_{e}^{V}(\vec{r}, \vec{k}) \ln f_{e}^{V}(\vec{r}, \vec{k})+\left[1-f_{e}^{V}(\vec{r}, \vec{k})\right] \ln \left[1-f_{e}^{V}(\vec{r}, \vec{k})\right]\right\} d^{3} k
$$




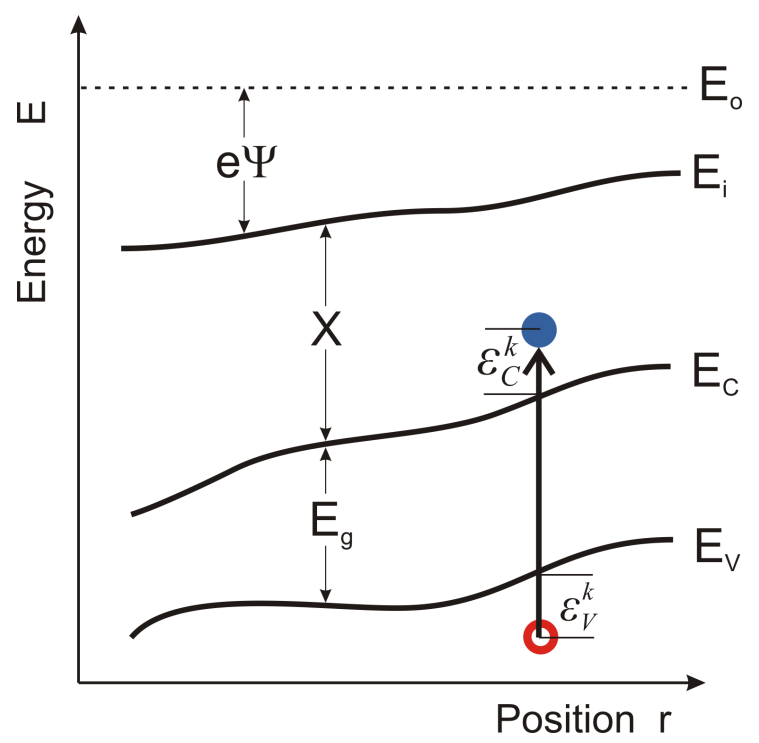

Figure 8. General one-dimensional two-band energy diagram of a semiconductor heterostructure defining variables of interest; $E$ represents total electron energy; $E_{0}$ is the reference level (we have assumed $E_{0}=0$ ); $\Psi$ is the electrostatic potential; $X$ is the electron affinity; $E_{i}$ is the local vacuum level (defined by Marshak and Vliet [2]); $E_{V}$ is the valence band (VB) edge; $E_{C}$ is the conductance band (CB) edge; and $E_{g}$ is the bandgap. The transition of the electron from energy level $E_{V}-\varepsilon_{V}^{k}$ in VB to energy level $E_{C}+\varepsilon_{C}^{k}$ in CB demands that the delivery of energy equals $E_{g}+\varepsilon_{C}^{k}+\varepsilon_{V}^{k}$. This energy is the sum of the energy of the created electron $-\chi+e \Psi+\varepsilon_{C}^{k}$ and the energy of the created hole $\chi+e \Psi+E_{g}+\varepsilon_{V}^{k}$.

\section{B. The Sources of Electron and Hole Entropy in Steady-State Conditions}

Both the electrons' energy and the holes' energy contain kinetic energy (see Appendix A) associated with the chaotic thermal particles' motion and the potential energy, determined by electrochemical potentials $\Phi$ (determined by the energies of band edges and chemical potentials). In thermal equilibrium, it is possible to apply the first law of thermodynamics. If we consider the densities of extensive variables (entropy and the number of electrons), the first law of thermodynamics for the differential of the electrons energy is expressed by Gibbs relation:

$$
d u_{e}=T d s_{e}+\Phi d n
$$

However, opportunely, the expression for the holes:

$$
d u_{h}=T d s_{h}-\Phi d p
$$

where $d u$ is the increment of energy density (subscript $e$ refers to electrons, subscript $h$ to holes), $s$, the density of entropy, $n$, the electron density (concentration), $p$, the hole concentration and $\Phi$, Fermi energy. Thus, the increment of density of the carriers' energy is caused by a change of the particles' density, the consecutive changing potential energy and the energy associated with the increment of the density of entropy. 
Now, we would like to analyze the structure of a semiconductor in steady-state conditions. We have used the postulate of local equilibrium. The first law of thermodynamics for the increments of electrons' and holes' density of energy changing locally in a semiconductor structure is expressed by:

$$
\begin{aligned}
& d u_{e}=T_{e} d s_{e}+\Phi_{n} d n \\
& d u_{h}=T_{h} d s_{h}-\Phi_{p} d p,
\end{aligned}
$$

where effective temperatures of electrons and holes ( $T_{e}$ and $T_{h}$, respectively) and quasi-electrochemical potentials (quasi-Fermi energies, $\Phi_{n}$ and $\Phi_{p}$ ) are defined locally by the relations:

$$
\left(\frac{\partial u_{e}}{\partial s_{e}}\right)_{\Phi_{n}}=T_{e} \quad\left(\frac{\partial u_{e}}{\partial n}\right)_{T_{e}}=\Phi_{n}
$$

and

$$
\left(\frac{\partial u_{h}}{\partial s_{h}}\right)_{\Phi_{p}}=T_{h} \quad\left(\frac{\partial u_{h}}{\partial p}\right)_{T_{h}}=-\Phi_{p} .
$$

Here, $\Phi_{n}$ and $\Phi_{p}$ are the electrochemical potential for CB electrons and CV electrons, respectively. The electrochemical potential for holes is equal to $-\Phi_{p}$.

Let us consider Equation (52). The first component is defined as thermal energy $d Q_{n}$. The second component includes the increment of potential energy $d u_{\Phi_{n}}$, determined by electrochemical potential. They are expressed by:

$$
d u_{e}=d Q_{n}+d u_{\Phi_{n}} .
$$

If there are flows of particles and energy, the balance equations for physical parameters are subject to a low of conservation and are expressed by:

$$
\frac{\partial x}{\partial t}+\nabla j_{x}=\left(G_{x}-R_{x}\right)
$$

where $x$ is the density of the considered extensive parameter, $j_{x}$ is the current density of $x, G_{x}$ is the generation rate and $R_{x}$ the recombination rate of the $x$ parameter. For $u_{e}$, we obtain the following balance equation:

$$
\frac{\partial u_{e}}{\partial t}=-\nabla j_{Q_{e}}+\left(G_{n}-R_{n}\right) \widetilde{\varepsilon}_{e}^{k}-\nabla\left(\Phi_{n} j_{e}\right)+\left(G_{n}-R_{n}\right) \Phi_{n}
$$

Here, $\widetilde{\varepsilon}_{e}^{k}$ is the average of the kinetic energy of electrons generated with the net generation rate $\left(G_{n}-R_{n}\right)$, and $j_{e}$, the density of the electron current (current of particles, no electric current). Similarly, as for electrons, the balance of energy density equation for holes, $u_{h}$, is expressed by:

$$
\frac{\partial u_{h}}{\partial t}=-\nabla j_{Q_{h}}+\left(G_{p}-R_{p}\right) \widetilde{\varepsilon}_{h}^{k}+\nabla\left(\Phi_{p} j_{h}\right)-\left(G_{p}-R_{p}\right) \Phi_{p} .
$$

Here, $j_{Q_{h}}$ is the density of the hole's heat current; $j_{h}$, the density of the hole's current (particles current); $G_{p}$, the density of the generation rate; $R_{p}$, the density of the hole's recombination rate; $u_{\Phi_{p}}$, the potential energy of the holes, defined by quasi-Fermi energy $\Phi_{p}$.

A was shown by Parrot [29], there is a simple relation between the density of thermal energy current and the density of entropy current:

$$
j_{Q_{n}}=T_{e} j_{s_{e}}=T_{e} s_{e} \bar{v}_{e}
$$


where $s_{e}$ is the density of the electron entropy and $\bar{v}_{e}$ is an average electron velocity. A similar equation for holes is expressed by [29]:

$$
j_{Q_{p}}=T_{h} j_{s_{h}}=T_{h} s_{h} \bar{v}_{h},
$$

where $s_{h}$ is the density of the hole entropy and $\bar{v}_{e}$ is an average hole velocity.

Entropy is not subject to the rules of conservation. Thus, it is difficult to drive an expression for the entropy balance equation. If we try to use the first law of thermodynamics, applied to the selected subsystem in the local equilibrium (Equation (52)), the increment of the entropy of electrons may be expressed by the increments of the particles' number and energy. It is expressed by the equation:

$$
\frac{\partial s_{e}}{\partial t}=\frac{1}{T_{e}} \frac{\partial u_{e}}{\partial t}-\frac{\Phi_{n}}{T_{e}} \frac{\partial n}{\partial t} .
$$

The balance equation for the electrons density (concentration) has the form:

$$
\frac{\partial n}{\partial t}=-\nabla j_{n}+\left(G_{n}-R_{n}\right)
$$

Using Equations (58), (60) and (63), Equation (62) can be converted to the form:

$$
\frac{\partial s_{e}}{\partial t}=-\frac{\nabla T_{e}}{T_{e}} j_{s}-\nabla j_{s}+\frac{1}{T_{e}}\left(G_{n}-R_{n}\right) \widetilde{\varepsilon}_{e}^{k}-\frac{\nabla \Phi_{n}}{T_{e}} j_{n} .
$$

Equation (64) has the form of Equation (57); thus, it is an equation of the entropy density balance. The source of the density of electron entropy is now given by the expression:

$$
\sigma_{s_{e}}=-\frac{\nabla \Phi_{n}}{T_{e}} j_{n}-\frac{\nabla T_{e}}{T_{e}} j_{s_{e}}+\frac{1}{T_{e}}\left(G_{n}-R_{n}\right) \widetilde{\varepsilon}_{e}^{k}
$$

In a steady state, the electrons' generation rate is equal to the rate of the electrons' recombination; thus, expression Equation (65) can be simplified to the form:

$$
\sigma_{s_{e}}=-\frac{\nabla \Phi_{n}}{T_{e}} j_{n}-\frac{\nabla T_{e}}{T_{e}} j_{s_{e}}
$$

Similar considerations for holes lead us to the expression for the source of the holes entropy in steady-state conditions:

$$
\sigma_{s_{h}}=\frac{\nabla \Phi_{p}}{T_{h}} j_{p}-\frac{\nabla T_{h}}{T_{h}} j_{s_{h}}
$$

The left side of Equation (66) one can express in two ways. In the first method, the electron current density and density of their entropy current are derived by the integration of electron velocity $\frac{\hbar \vec{k}}{m_{e}^{*}}$ inside the Brillouin zone (BZ) as below.

$$
\begin{aligned}
\sigma_{s_{e}}= & -\frac{1}{4 \pi^{3}} \frac{\nabla \Phi_{n}}{T_{e}} \int_{B Z} f_{e}^{C}(\vec{r}, \vec{k}) \frac{\hbar \vec{k}}{m_{e}^{*}} d^{3} k \\
& +\frac{k_{B}}{4 \pi^{3}} \frac{\nabla T_{e}}{T_{e}} \int_{B Z}\left\{f_{e}^{C}(\vec{r}, \vec{k}) \ln f_{e}^{C}(\vec{r}, \vec{k})+\left[1-f_{e}^{C}(\vec{r}, \vec{k})\right] \ln \left[1-f_{e}^{C}(\vec{r}, \vec{k})\right]\right\} \frac{\hbar \vec{k}}{m_{e}^{*}} d^{3} k
\end{aligned}
$$

In the second method, the electron current density, as well as the density of entropy current are expressed by the electron drift velocity $\bar{v}_{d}^{e}$ :

$$
\bar{v}_{d}^{e}=\bar{\tau}_{e} \frac{F_{e}}{m_{e}^{*}},
$$


where $\bar{\tau}_{e}$ is the average electron relaxation time, $m_{e}^{*}$ the effective electron mass and $F_{e}$ is the electron driving force expressed by:

$$
F_{e}=-\nabla \Phi_{n}-\Sigma_{e} \nabla T_{e}
$$

where $\Sigma_{e}$ is Seebeck's coefficient for electrons. As was shown in Appendix C, $\Sigma_{e}=\frac{s_{e}}{n}$.

The relaxation time in semiconductors is determined by some different scattering mechanisms [13]. The average relaxation time [5] is expressed by:

$$
\bar{\tau}_{e}=\frac{\frac{1}{4 \pi^{3}} \int_{B Z} f_{e}^{C} \tau_{e}\left(\varepsilon_{e}^{k}(\vec{k})\right) \varepsilon_{e}^{k}(\vec{k}) d^{3} k}{\frac{1}{4 \pi^{3}} \int_{B Z} f_{e}^{C} \varepsilon_{e}^{k}(\vec{k}) d^{3} k}=\frac{\frac{1}{4 \pi^{3}} \int_{B Z} f_{e}^{C} \tau_{e}\left(\varepsilon_{e}^{k}(\vec{k})\right) \varepsilon_{e}^{k}(\vec{k}) d^{3} k}{\frac{3}{2} n k_{B} T_{e}}
$$

where $\varepsilon_{e}^{k}(\vec{k})$ is the kinetic energy of the electron with quasi-momentum $\hbar \vec{k}$.

Now, Equation (66) can be expressed in the form:

$$
\begin{aligned}
\sigma_{s_{e}} & =\frac{T_{e}}{4 \pi^{3}}\left(\frac{\nabla \Phi_{n}}{T_{e}}\right)\left(\frac{\nabla \Phi_{n}}{T_{e}}\right) \int_{B Z} f_{e}^{C}(\vec{r}, \vec{k}) \frac{\bar{\tau}_{e}}{m_{e}^{*}} d^{3} k \\
& -\frac{k_{B} T_{e}}{4 \pi^{3}}\left(\frac{\nabla \Phi_{n}}{T_{e}}\right)\left(\frac{\nabla T_{e}}{T_{e}}\right) \int_{B Z}\left\{f_{e}^{C}(\vec{r}, \vec{k}) \ln f_{e}^{C}(\vec{r}, \vec{k})+\left[1-f_{e}^{C}(\vec{r}, \vec{k})\right] \ln \left[1-f_{e}^{C}(\vec{r}, \vec{k})\right]\right\} \frac{\bar{\tau}_{e}}{m_{e}^{*}} d^{3} k \\
& -\frac{k_{B} T_{e}}{4 \pi^{3}}\left(\frac{\nabla T_{e}}{T_{e}}\right)\left(\frac{\nabla \Phi_{n}}{T_{e}}\right) \int_{B Z}\left\{f_{e}^{C}(\vec{r}, \vec{k}) \ln f_{e}^{C}(\vec{r}, \vec{k})+\left[1-f_{e}^{C}(\vec{r}, \vec{k})\right] \ln \left[1-f_{e}^{C}(\vec{r}, \vec{k})\right]\right\} \frac{\bar{\tau}_{e}}{m_{e}^{*}} d^{3} k \\
& +\frac{k_{B}}{4 \pi^{3}}\left(\frac{\nabla T_{e}}{T_{e}}\right)\left(\frac{\nabla T_{e}}{T_{e}}\right) \int_{B Z}\left\{f_{e}^{C}(\vec{r}, \vec{k}) \ln f_{e}^{C}(\vec{r}, \vec{k})+\left[1-f_{e}^{C}(\vec{r}, \vec{k})\right] \ln \left[1-f_{e}^{C}(\vec{r}, \vec{k})\right]\right\} \frac{\bar{\tau}_{e}}{m_{e}^{*}} d^{3} k \\
& \cdot \frac{k_{B}}{4 \pi^{3} n} \int_{B Z}\left\{f_{e}^{C}(\vec{r}, \vec{k}) \ln f_{e}^{C}(\vec{r}, \vec{k})+\left[1-f_{e}^{C}(\vec{r}, \vec{k})\right] \ln \left[1-f_{e}^{C}(\vec{r}, \vec{k})\right]\right\} d^{3} k
\end{aligned}
$$

Similar considerations for holes lead to two different forms of expressions for $\sigma_{s_{h}}$ :

$$
\begin{aligned}
\sigma_{s_{h}}= & \frac{1}{4 \pi^{3}} \int_{Z B}\left[1-f_{e}^{V}(\vec{r}, \vec{k})\right] \frac{\hbar \vec{k}}{m_{h}^{*}} \frac{\nabla \Phi_{h}}{T_{h}} d^{3} k \\
& +\frac{k_{B}}{4 \pi^{3}} \int_{Z B}\left\{f_{e}^{V}(\vec{r}, \vec{k}) \ln f_{e}^{V}(\vec{r}, \vec{k})+\left[1-f_{e}^{V}(\vec{r}, \vec{k})\right] \ln \left[1-f_{e}^{V}(\vec{r}, \vec{k})\right]\right\} \frac{\hbar \vec{k}}{m_{h}^{*}} \frac{\nabla \Phi_{h}}{T_{h}} d^{3} k
\end{aligned}
$$

and:

$$
\begin{aligned}
\sigma_{s_{h}} & =\frac{T_{h}}{4 \pi^{3}}\left(\frac{\nabla \Phi_{p}}{T_{h}}\right)\left(\frac{\nabla \Phi_{p}}{T_{h}}\right) \int_{B Z}\left(1-f_{e}^{V}(\vec{r}, \vec{k})\right) \frac{\bar{\tau}_{h}}{m_{h}^{*}} d^{3} k \\
& +\frac{k_{B} T_{h}}{4 \pi^{3}}\left(\frac{\nabla \Phi_{p}}{T_{h}}\right)\left(\frac{\nabla T_{h}}{T_{h}}\right) \int_{B Z}\left\{f_{e}^{V}(\vec{r}, \vec{k}) \ln f_{e}^{V}(\vec{r}, \vec{k})+\left[1-f_{e}^{V}(\vec{r}, \vec{k})\right] \ln \left[1-f_{e}^{V}(\vec{r}, \vec{k})\right]\right\} \frac{\bar{\tau}_{h}}{m_{h}^{*}} d^{3} k \\
& +\frac{k_{B} T_{h}}{4 \pi^{3}}\left(\frac{\nabla T_{h}}{T_{h}}\right)\left(\frac{\nabla \Phi_{p}}{T_{h}}\right) \int_{B Z}\left\{f_{e}^{V}(\vec{r}, \vec{k}) \ln f_{e}^{V}(\vec{r}, \vec{k})+\left[1-f_{e}^{v}(\vec{r}, \vec{k})\right] \ln \left[1-f_{e}^{V}(\vec{r}, \vec{k})\right]\right\} \frac{\bar{\tau}_{h}}{m_{h}^{*}} d^{3} k \\
& +\frac{k_{B} T_{h}}{4 \pi^{3}}\left(\frac{\nabla T_{h}}{T_{h}}\right)\left(\frac{\nabla T_{h}}{T_{h}}\right) \int_{B Z}\left\{f_{e}^{V}(\vec{r}, \vec{k}) \ln f_{e}^{V}(\vec{r}, \vec{k})+\left[1-f_{e}^{V}(\vec{r}, \vec{k})\right] \ln \left[1-f_{e}^{V}(\vec{r}, \vec{k})\right]\right\} \frac{\bar{\tau}_{h}}{m_{h}^{*}} d^{3} k \\
& +\frac{k_{B}}{4 \pi^{3} p} \int_{B Z}\left\{f_{e}^{V}(\vec{r}, \vec{k}) \ln f_{e}^{V}(\vec{r}, \vec{k})+\left[1-f_{e}^{V}(\vec{r}, \vec{k})\right] \ln \left[1-f_{e}^{V}(\vec{r}, \vec{k})\right]\right\} d^{3} k
\end{aligned}
$$




\section{The Transport Coefficients of Semiconductor Heterostructures in Steady-State Conditions}

Forces acting on electric carriers in a semiconductor heterostructure are caused by gradients of quasi-Fermi energies or the gradient of temperature. To determine them, one has to find the kinetic coefficients by which these gradients have to be multiplied. Denoting these gradients by $\nabla \Phi$ and $\nabla \Psi$, respectively, the usual results, as found for example in the paper of Marshak and van Vliet [2], are:

$$
\begin{aligned}
& j^{e l}=L_{11} \nabla \Phi_{n}+L_{12} \nabla T \\
& j_{s_{e}}=L_{21} \nabla \Phi_{n}+L_{22} \nabla T .
\end{aligned}
$$

The Onsager conductances (L) are band integrals, such as $L_{11}$ and $L_{12}$ for electrical conductance in a conduction band. The band integrals are found in the classical article by Marshak and van Vliet [2] and more succinctly in the latter's recent book [30]. Lindefelt [3] approximated their results by using a "mean" energy $\langle E\rangle$ ( $c f$. his Equation (12)); thus, for Equation (75), he wrote,

$$
j^{e l}=n \mu_{e}\left\{\nabla \Phi_{n}+\left[\left(\langle E\rangle-\Phi_{n}\right) / T_{e}\right] \nabla T_{e}\right\} .
$$

Here, $j^{e l}$ is the electron electric current, and $\mu_{e}$ is the electron mobility.

We also note that the entropy per particle for the conduction band is just given by:

$$
\frac{s_{e}}{n}=\left(\langle E\rangle-\Phi_{n}\right) / T_{e}
$$

Thus, relation Equation (77) reads:

$$
j^{e l}=n \mu_{e}\left\{\nabla \Phi_{n}+\frac{s_{e}}{n} \nabla T_{e}\right\} .
$$

This way, $\frac{s_{e}}{n}=\Sigma_{e}$ is Seebeck's coefficient.

On the other hand, by multiplying $\sigma_{s_{e}}$ (relation Equation (66)) by $T_{e}$, we obtain the heat energy generated in a unit of volume and a unit of time, that is $\epsilon_{e}^{k i n}$; the kinetic energy of CB electrons (in $\mathrm{J} \mathrm{m}^{-3} \mathrm{~s}^{-1}$ ) reads:

$$
\frac{\partial q_{e}}{\partial t}=\sigma_{s_{e}} T_{e}=-j_{s_{e}} \nabla T_{e}-j_{e} \nabla \Phi_{n}=-s_{e} \bar{v}_{e} \nabla T_{e}-n \bar{v}_{e} \nabla \Phi_{n}=\frac{\partial \varepsilon_{e}^{k}}{\partial t} .
$$

On the other hand, the time derivative of electron kinetic energy in a unit of volume is equal to:

$$
\frac{d \varepsilon_{e}^{k}}{d t}=\bar{v}_{e} F_{e}=\bar{v}_{e}\left(-n \nabla \Phi_{n}-s_{e} \nabla T_{e}\right)
$$

where $F_{e}$ is the mean force acting on all conduction-band electrons in a unit volume. Therefore, now, the mean force acting on a single electron reads:

$$
\frac{F_{n}}{n}=-\nabla \Phi_{n}-\frac{s_{e}}{n} \nabla T_{e} .
$$

The transport coefficient $\frac{s_{e}}{n}=\Sigma_{e}$ is here recognized as the Seebeck coefficient for electrons.

Similarly as for electrons, by multiplying $\sigma_{s_{h}}$ (relation Equation (67)) by $T_{h}$, we obtain the heat energy generated in a unit of volume and a unit of time, that is the kinetic energy of holes (in $\mathrm{J} \mathrm{m}^{-3} \mathrm{~s}^{-1}$ ). This leads to the expression of the mean force acting on all holes in a unit volume:

$$
F_{h}=p \nabla \Phi_{p}-s_{h} \nabla T_{h}
$$


and, next, to the expression of the average force acting on a single hole.

$$
\frac{F_{h}}{p}=\nabla \Phi_{p}-\frac{s_{h}}{p} \nabla T_{h}
$$

Seebeck's coefficient for holes is found as $\Sigma_{h}=\frac{s_{h}}{p}$.

\section{References}

1. Van Vliet, K.M.; Marshak, A.H. Conduction current and generalized einstein relation for degenerate semiconductors and metals. Phys. Stat. Sol. 1976, 78, 501-517.

2. Marshak, A.H.; van Vliet, K.M. Electrical current and carrier density in degenerate materials with nonuniform band structure. Proc. IEEE 1984, 72, 148-162.

3. Lindefelt, U. Current-density relation for nonisotermal modeling of degenerate heterostructure devices. J. Appl. Phys. 1994, 75, 958-966.

4. Parrot, J.E. Thermodynamic theory of transport processes in semiconductors. EEE Trans. Electron Devices 1996, 43, 809-826.

5. Lundstrom, M. Fundamentals of Carrier Transport; Addison-Wesley Publishing Company, Inc.: Boston, MA, USA, 1990; Volume 10, page 133.

6. Prigogine, I. Introduction to Thermodynamics of Irreversible Processes; John Wiley: Hoboken, NJ, USA, 1956.

7. De Groot, S.R.; Mazur, P. Non-equilibrium Thermodynamics; Wiley: New York, NY, USA, 1962.

8. Gyarmati, I. Non-equilibrium Field Theory and Variational Principles; Springer Verlag: Berlin, Germany, 1970.

9. Glansdorf, P.; Prigogine, I. Thermodynamic Theory of Structure, Stability and Fluctuations; Wiley Interscience: Hoboken, NJ, USA, 1971.

10. Prigogine, I. Modération et transformations irreversibles des systemes ouverts. Bull. Cl. Sci. Acad. R. Belg. 1945, 31, 600-606.

11. Prigogine, I. Étude Thermodynamique des Phenoménes Irreversibles; Desoer: Paris, France, 1947.

12. Lundstrom, M. Fundamentals of Carrier Transport; Addison-Wesley Publishing Company, Inc.: Boston, MA, USA, 1990; Volume 10, p. 103.

13. Nag, B.R. Electron Transport in Compound Semiconductors; Springer Verlag: Berlin, Germany, 1980.

14. Gyarmati, I. On the relation between Onsager's and Prigogine's principles. Zeitschrift für Physikalische Chemie 1967, 234, 371-378.

15. Gyarmati, I. On the governing principle of dissipative processes and its extension to non-linear problems. Ann. Phys. 1969, 23, 253-378.

16. Verhas, J. Gyarmati's variational principle of disipative processes. Entropy 2014, 16, 2364-2383.

17. Markus, F.; Gambar, K. A variational principle in thermodynamics. J. Non-Equilib. Thermodyn. 1991, 16, 27-32.

18. Markus, F.; Gambar, K. Hamilton's canonical equations and the entropy production. J. Non-Equilib. Thermodyn. 1992, 18, 288-292.

19. Markus, F.; Gambar, K. Comments on the relationship between extended thermodynamics and the wave approach to thermodynamics. J. Non-Equilib. Thermodyn. 1989, 14, 355-362. 
20. Onsager, L. Reciprocal relations on irreversible processes. I. Phys. Rev. 1931, 37, 405-426.

21. Onsager, L. Reciprocal relations on irreversible processes. II. Phys. Rev. 1931, 38, 2265-2279.

22. Onsager, L.; Maclup, S. Fluctuations and irreversible processes. Phys. Rev. 1953, 91, 1505-1512.

23. Farkas, H. The reformulation of the Gyarmati principle in a generalized picture. Zeitschrift für Physikalische Chemie 1968, 239, 124-132.

24. Bhattacharya, D.K. A variational principle for thermodynamical waves. Ann. Phys. 1983, 39, 325-332.

25. Verhas, J. Gayrmati's variational principle of dissipative processes. In Proceedings of the 12th Joint European Thermodynamics Conference, JETC 2013, Brescia, Italy, 1-5 July 2013; pp. 127-132.

26. Capper, P., Ed. Properties of Narrow Gap Cadmium-based Compounds; The Institution of Engineering and Technology: London, UK, 1994.

27. Jóźwikowska, A. Numerical solution of the nonlinear Poisson equation for semiconductor devices of a diffusion-equation finite difference scheme. J. Appl. Phys. 2008, 104, 063715.

28. Landau, L.D.; Lifshitz, E.M. Statistical Physics, 3rd ed.; Butterworth-Heinemann: Oxford, UK, 1980.

29. Parrott, J.E. Transport theory of semiconductor energy conversion. J. Appl. Phys. 1982, 53, 9105-9111.

30. Van Vliet, K. Equilibrium and Non-equilibrium Statistical Mechanics; World Scientific: Toh Tuck, Singapore, 2010.

(c) 2015 by the authors; licensee MDPI, Basel, Switzerland. This article is an open access article distributed under the terms and conditions of the Creative Commons Attribution license (http://creativecommons.org/licenses/by/4.0/). 